\title{
Low-Order Stochastic Mode Reduction for a Realistic Barotropic Model Climate
}

\author{
Christian Franzke, Andrew J. Majda, And Eric Vanden-Eijnden \\ Courant Institute of Mathematical Sciences, New York University, New York, New York
}

(Manuscript received 24 March 2004, in final form 12 October 2004)

\begin{abstract}
This study applies a systematic strategy for stochastic modeling of atmospheric low-frequency variability to a realistic barotropic model climate. This barotropic model climate has reasonable approximations of the Arctic Oscillation (AO) and Pacific/North America (PNA) teleconnections as its two leading principal patterns of low-frequency variability. The systematic strategy consists first of the identification of slowly evolving climate modes and faster evolving nonclimate modes by use of an empirical orthogonal function (EOF) decomposition. The low-order stochastic climate model predicts the evolution of these climate modes a priori without any regression fitting of the resolved modes. The systematic stochastic mode reduction strategy determines all correction terms and noises with minimal regression fitting of the variances and correlation times of the unresolved modes. These correction terms and noises account for the neglected interactions between the resolved climate modes and the unresolved nonclimate modes. Loworder stochastic models with only four resolved modes capture the statistics of the original barotropic model modes quite well. A budget analysis establishes that the low-order stochastic models are dominated by linear dynamics and additive noise. The linear correction terms and the additive noise stem from the linear coupling between resolved and unresolved modes, and not from nonlinear interactions between resolved and unresolved modes as assumed in previous studies.
\end{abstract}

\section{Introduction}

The understanding of low-frequency variability on time scales of more than 10 days to one season has attracted a lot of attention. Most of the midlatitude low-frequency variability can be described by a few recurring persistent teleconnection patterns (Wallace and Gutzler 1981; Barnston and Livezey 1987). These patterns have a strong impact on surface climate and are related to global warming (Marshall et al. 2001; Thompson and Wallace 2001). The atmospheric circulation shows a large range of variability both on spatial and temporal scales. This variability ranges from these persistent large-scale teleconnection patterns, to synoptic scale waves with a time scale of a few days, and rather small scale boundary layer processes acting on an hourly time scale. Due to the nonlinear character of the equations of motion, all of these processes are dynamically coupled. It is quite interesting for climate studies to develop systematic low-order models for the largescale teleconnection patterns of the atmosphere. One needs to properly account for the coupling with shorter time scale unresolved processes and this is known as the

Corresponding author address: Dr. Christian Franzke, Courant Institute of Mathematical Sciences, New York University, 251 Mercer Street, New York, NY 10012.

E-mail: franzke@cims.nyu.edu closure problem. The issue of whether the large-scale low-frequency atmospheric variability can be modeled systematically by a low-dimensional stochastic dynamical system is the main topic of this paper.

In numerical atmospheric models the atmospheric fields are represented on a particular grid or are expanded in spherical harmonics, in so-called spectral models. All features smaller than the grid size or smaller than the smallest wavelength are neglected. Parameterizations are used to account for these neglected processes and their interaction with the resolved scales. Highly truncated spectral models (Charney and DeVore 1979; Reinhold and Pierrehumbert 1982; Legras and Ghil 1985) have been developed to give insight into the dynamics of low-frequency variability and atmospheric regime behavior, but they exhibit only very idealized flow patterns.

This raises the question if the descriptions used in numerical models are optimal choices in describing large-scale atmospheric features. Decomposing the atmospheric fields more directly in terms of the recurring teleconnection patterns could have advantages. Possible choices of better basis functions are empirical orthogonal functions (EOF) (Schubert 1985; Selten 1995; Achatz and Branstator 1999) and principal interaction patterns (PIP) (Hasselmann 1988; Kwasniok 1996, 2004). Truncated EOF models show a climate drift due to the neglected interactions with the unresolved 
modes. Selten (1995) and Achatz and Branstator (1999) parameterize these neglected interactions by a linear damping, whose strength is determined empirically. A more powerful tool to extract the dynamics of a system are PIPs (Kwasniok 1996, 2004). The calculation of PIPs takes into account the dynamics of the model for which one tries to find an optimal basis and also often involves ad hoc closure through linear damping and an ansatz for nonlinear interactions. Crommelin and Majda (2004) compare different optimal bases. They find that models based on PIPs are superior to models based on EOFs and optimal persistence patterns (OPP; Delsole 2001). On the other hand, they also point out that the determination of PIPs can show sensitivities regarding the calculation procedure, at least for some loworder atmospheric dynamical systems with regime transitions. This feature makes PIPs possibly a less attractive basis.

The low-order models described above are based on physical principles combined with an ad hoc closure principle involving damping. They take the governing system of equations and project them on more or less suitable basis functions. Another method is to determine the model by empirical fitting procedures. This approach linearizes the equations of motion around some basic state, determines the linear operator empirically, and adds some kind of forcing: this forcing can be random and is considered as taking account of the neglected nonlinear processes (Newman et al. 1997; Whitaker and Sardeshmukh 1998; Zhang and Held 1999; Winkler et al. 2001) or represents external forcing like tropical heating (Branstator and Haupt 1998). To ensure stability of these linear models these studies add linear damping according to various ad hoc principles. There is a recent survey of such strategies (Delsole 2004).

Another approach is to fit simple stochastic models to scalar teleconnection indices. Wunsch (1999) argues that the North Atlantic Oscillation (NAO) time series cannot be easily distinguished from a random stationary process. This is confirmed by Feldstein (2000), who concludes that the NAO is a Markov process with an $e$-folding time scale of about 10 days by fitting a firstorder autoregressive process. Stephenson et al. (2000) find that interannual variations of the NAO have a broadband spectrum, which is close to being white noise by analyzing monthly mean data. But they also present evidence for long-range dependencies by fitting a fractional differenced model. Percival et al. (2001) fit a first-order autoregressive and a fractionally differenced model to the North Pacific (NP) index. Both models show that the NP index exhibits significant correlations and also have a long tail of small but positive correlations for long lags. But their statistical tests cannot distinguish the superiority of one model over the other.

All these previous studies show encouraging results but have also their disadvantages in other respects.
Majda et al. (1999, 2001, 2002, 2003; collectively hereafter MTV) provide a systematic framework for how to account for the effect of the unresolved degrees of freedom on the resolved modes. The stochastic mode reduction strategy put forward in that work predicts all deterministic and stochastic correction terms. It has been applied and tested on a wide variety of simplified models and examples. The idealized models where the procedure has been tested, include those with trivial climates (MTV02), periodic orbits or multiple equilibria (MTV03), and heteroclinic chaotic orbits coupled to a deterministic bath of modes satisfying the truncated Burgers equation (Majda and Timofeyev 2000, 2004); the MTV procedure has been validated in these examples even when there is little separation of time scales between resolved and unresolved modes. To investigate the influence of topographic stress on the angular momentum budget, they applied this strategy to idealized flow over topography and derived a nonlinear reduced stochastic model with multiplicative noises (MTV03). This stochastic model turns out to be superior compared to a standard linear model with damping and white noise forcing. Also the explicit assumptions of varying the correlation times between the resolved modes and the unresolved modes in the MTV procedure for the climate model was checked explicitly (MTV03) by varying the topographic height. Here a seamless framework of the systematic stochastic mode reduction strategy from the MTV papers is developed for direct implementation in complex geophysical models. This seamless framework makes the practical implementation of the MTV procedure simpler with the same reduced stochastic equations for the unresolved modes. The mode reduction procedure is applied to a realistic barotropic model climate. We present results for the resulting low-order stochastic climate model with as little as only two resolved modes. The basis functions for this model are the dominant teleconnection patterns of the barotropic model climate. In section 2, we present the barotropic model and its climate. Section 3 describes the seamless MTV framework with details in an appendix, and section 4 gives the results of the reduced stochastic model. This paper closes with a summary and conclusions.

\section{Barotropic model climate}

\section{a. Barotropic model}

In this study a standard spectral barotropic model on the sphere is used with realistic orography. This model is forced with a forcing that was calculated from observations in order to get a realistic climatological mean state and low-frequency variability. The model equation is given by the barotropic vorticity equation

$$
\frac{\partial \zeta}{\partial t}=-J(\psi, \zeta+f+h)-\frac{\zeta}{\tau}+D \Delta^{3} \zeta+F,
$$


where $\zeta$ is the relative vorticity, $f=2 \Omega \sin \theta$ stands for the Coriolis parameter, $\Omega$ is the angular velocity of the sphere, $h$ is the topography; $\tau$ is the Ekman damping coefficient, $D$ the coefficient of the scale-selective damping, and $F$ is an external time-independent forcing (see appendix A). The equation has been nondimensionalized using the radius of the earth as unit of length and the inverse of the angular velocity of the earth as unit of time. The Jacobi operator $J(a, b)$ is defined by

$$
J(a, b)=\left(\frac{\partial a}{\partial \lambda} \frac{\partial b}{\partial \mu}-\frac{\partial a}{\partial \mu} \frac{\partial b}{\partial \lambda}\right)
$$

where $\mu$ denotes the sine of the latitude $\theta$, and $\lambda$ the longitude. The nondimensional orography $h$ is related to the realistic orography $h^{\prime}$ by $h=A_{0} h^{\prime} / H$, where $A_{0}=0.2$ is a factor that determines the strength of the surface wind that blows across the orography, and $H=$ $10 \mathrm{~km}$, a scale height (see Selten 1995). The model is truncated at T21. By restricting the spectral model to modes with zonal wavenumber plus total wavenumber being even, a model of hemispheric flow is obtained with a total number of 231 variables. The model has been integrated for $10^{5}$ days after a spinup period of 1000 days, using a fourth-order Adams-Bashforth time step scheme with a 45-min time step. The Ekman damping time scale is set to 15 days and the strength of the scale selective damping is such that wavenumber 21 is damped at a time scale of 3 days.

\section{b. Climatology}

The time mean streamfunction and the standard deviation are shown in Fig. 1. The simulated climate is different from the observations in some respects. The simulated ridges on the west coasts of North America and Europe are more pronounced than in the observations (Fig. 1a). The magnitude of the simulated variability is comparable with the observations (Fig. 1b). The simulated variability captures the maxima over the North Atlantic in the right location and amplitude. The observed maxima over the North Pacific is split in two: one is shifted southwestward and the other northwestward. But by considering all processes that the model lacks due to its barotropic nature (e.g., baroclinic instability, tropical convection, ENSO) and its coarse resolution (T21), the model climate is reasonably close to the observations. For the purpose here of testing the systematic stochastic mode reduction strategy the model climate is close enough to the observations.

EOFs of the streamfunction data are calculated. For the calculation, the time mean is subtracted from the data; the norm chosen is the kinetic energy norm in order to preserve nonlinear symmetries in the dynamics (see appendix B; Kwasniok 1996, 2004). The first EOF is characterized by a center of action over the Arctic that is surrounded by a zonal symmetric structure in midlatitudes (Fig. 2a). This pattern bears resemblance to the Arctic Oscillation/Northern Hemisphere Annular Mode (AO/NAM) (Thompson and Wallace 1998). This pattern explains about $14 \%$ of the total variance. The second EOF pattern is displayed in Fig. $2 \mathrm{~b}$ and resembles qualitatively the Pacific/North America (PNA) pattern (Wallace and Gutzler 1981). It consists of a meridional dipole over the Pacific Ocean at the end of the Pacific subtropical jet and two further centers of action over Canada and Florida. But it misses the center of action over the North Atlantic (Wallace and Thompson 2002). This pattern explains about $10 \%$ of a)

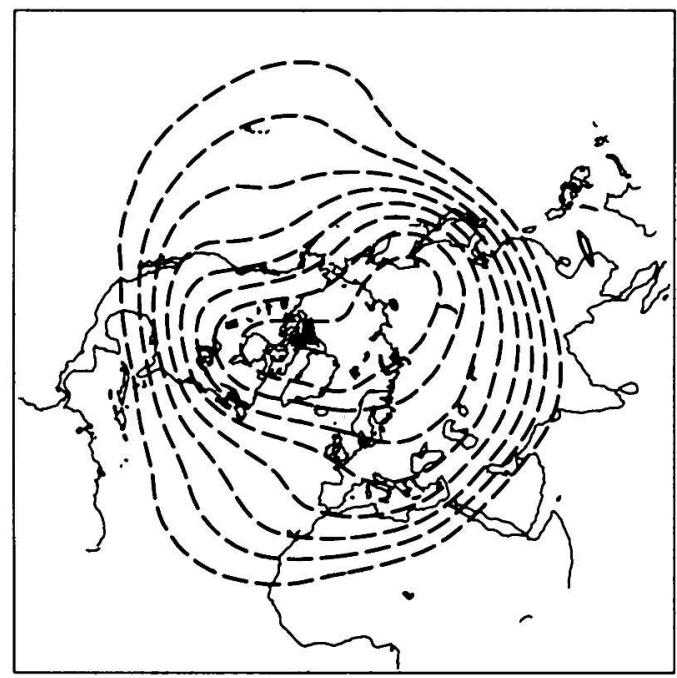

b)

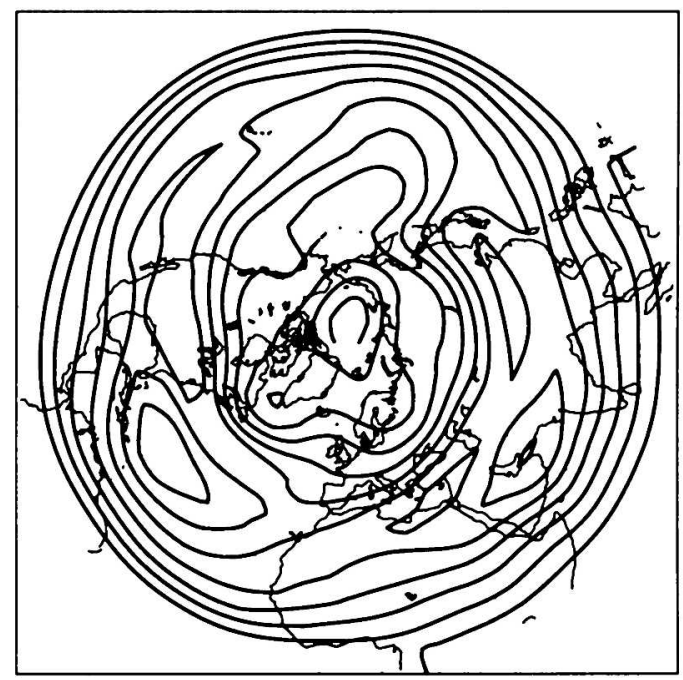

FIG. 1. (a) Climatological mean of T21 barotropic model (contour interval is $1.5 \times 10^{7} \mathrm{~m}^{2} \mathrm{~s}^{-1}$ ). (b) Standard deviation of low-pass filtered streamfunction of T21 barotropic model (contour interval is $2 \times 10^{6} \mathrm{~m}^{2} \mathrm{~s}^{-1}$ ). 
a)

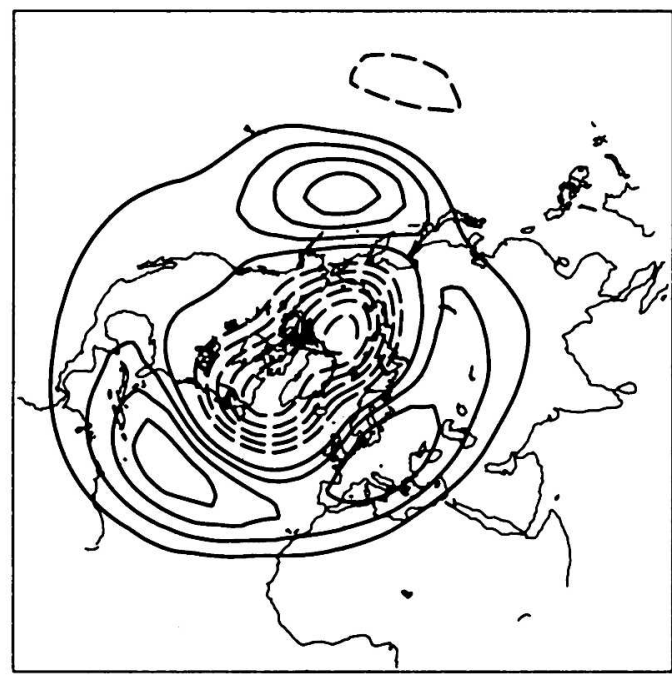

b)

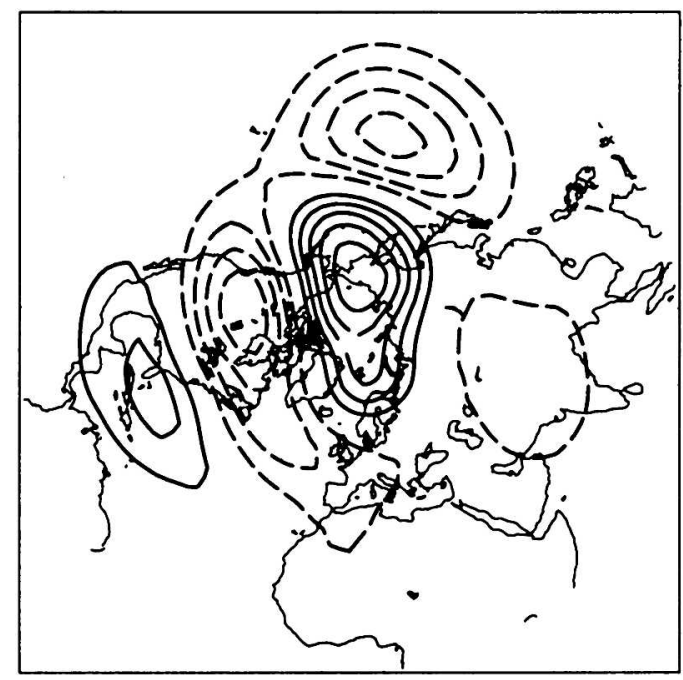

FIG. 2. Kinetic energy norm EOF: (a) EOF1 and (b) EOF2.

the total variance. The successive EOF patterns become increasingly small scale and do not correspond to well-known teleconnection patterns found in observational data. The cumulative explained variance of the first seven EOFs is more than $50 \%$ of the total variance (Fig. 3).

The autocorrelation functions for the first seven kinetic energy norm EOFs are displayed in Fig. 4. Some of the autocorrelation functions, such as modes $1,4,6$, and 7 , show only weak oscillations or no oscillations at all. While others, such as modes 2, 3, and 5, have stronger oscillations. The time scale set by the autocorrelation function is an important measure in this study because for the development of a stochastic climate model we have to make the truncation between slow and fast modes. The autocorrelation time scale, defined as the

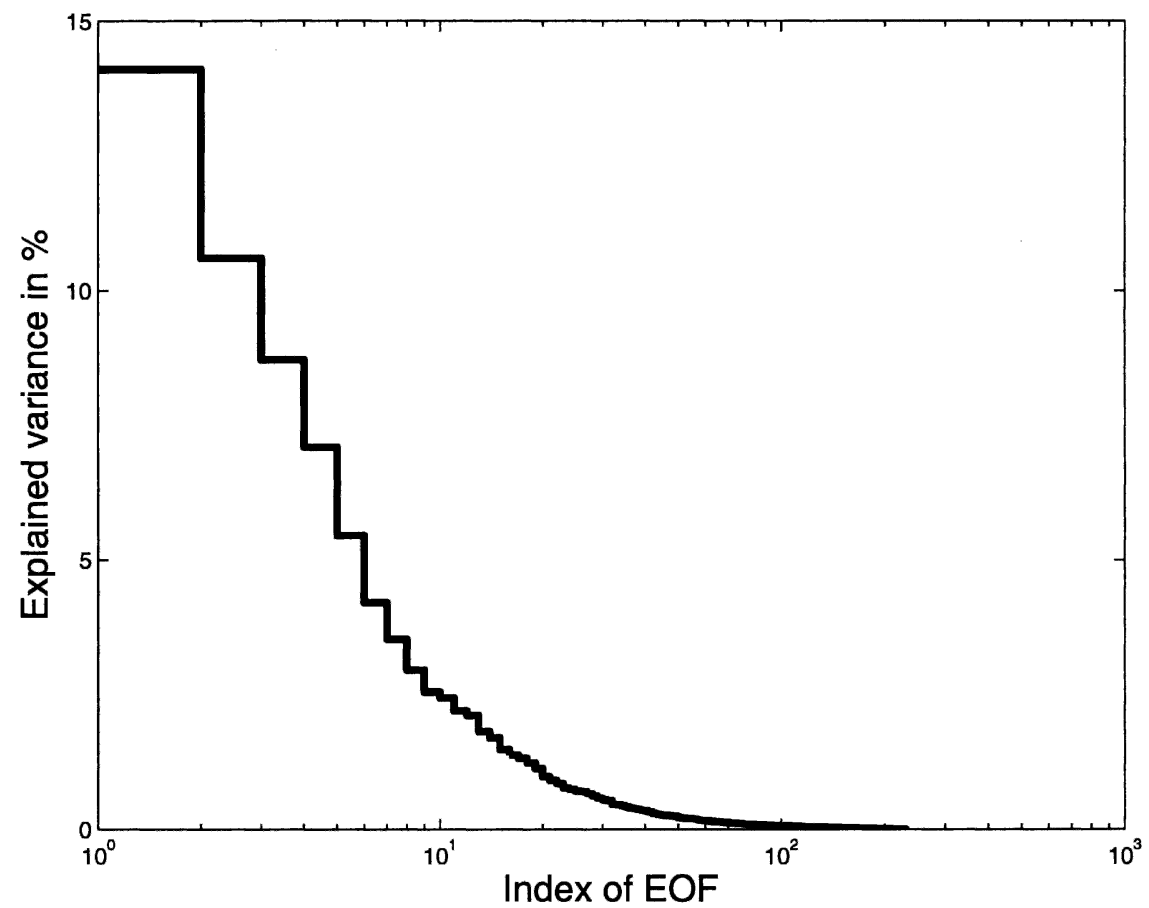

FIG. 3. Spectrum of the explained variances. 
a)

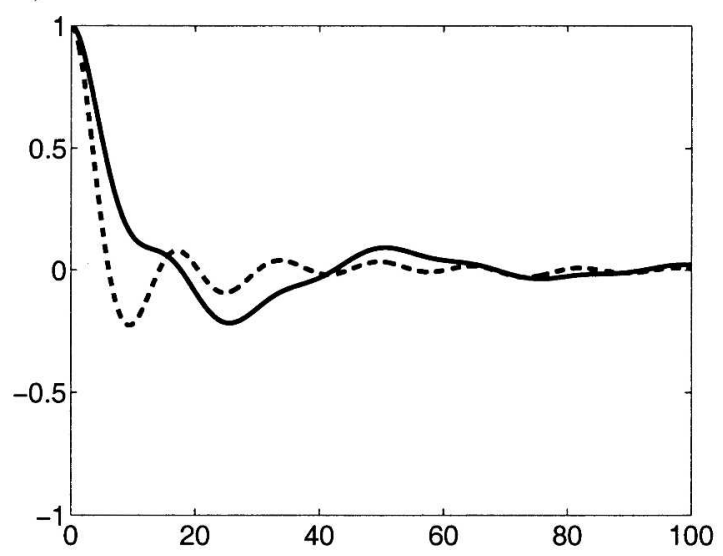

c)

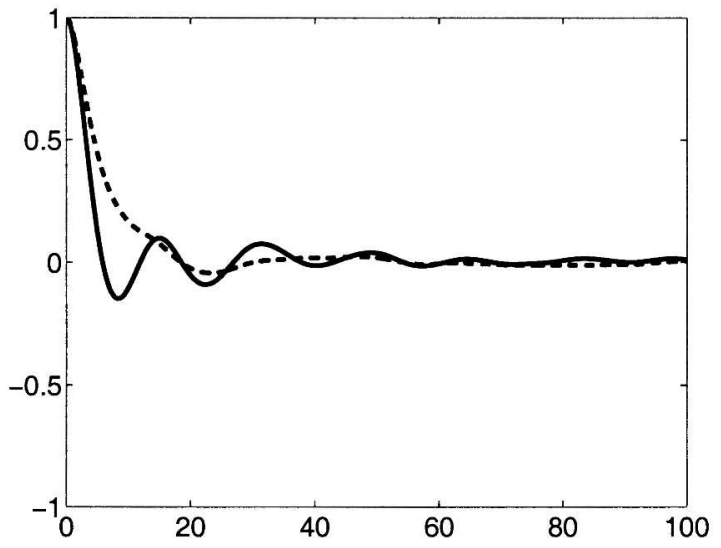

b)

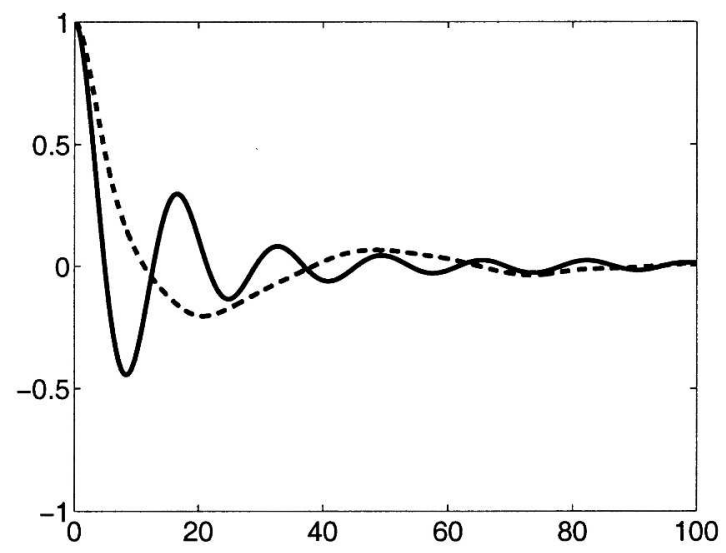

d)

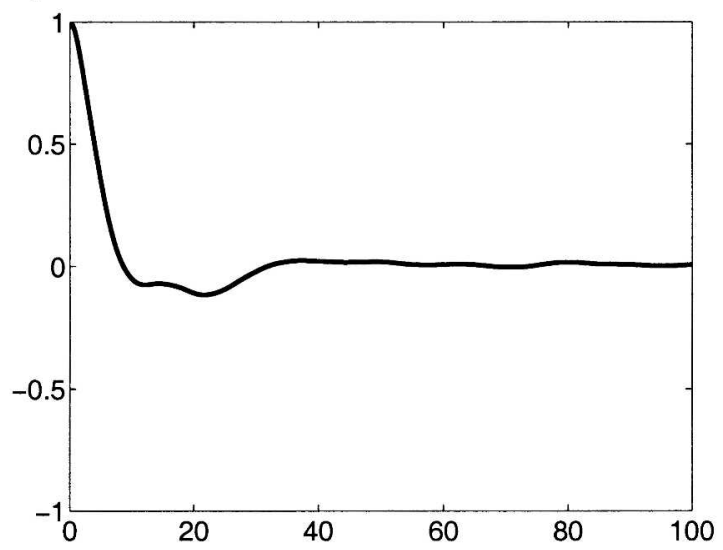

FIG. 4. Autocorrelation function of the amplitude of the kinetic energy norm EOFs of the barotropic model: (a) EOF1 (solid line) and EOF2 (dashed line), (b) EOF3 (solid line) and EOF4 (dashed line), (c) EOF5 (solid line) and EOF6 (dashed line), and (d) EOF7 (solid line).

integral over the absolute value of the autocorrelation function is

$$
T_{k}(s)=\frac{\left\langle a_{k}(t) a_{k}(t+s)\right\rangle}{\left\langle a_{k}^{2}\right\rangle},
$$

where $k$ indicates the $k$ th $\mathrm{EOF}$ mode, $s$ the time lag, and $\langle\cdot\rangle$ denotes a time average; $T_{k}(s)$ indicates how slow or fast a variable loses its memory. In Fig. 5 we show the autocorrelation time scale for all EOF modes. The first remarkable feature of this plot is that the decay of the autocorrelation time scale is not a monotonic function of the index of the EOF modes. Mode 1 has the slowest decay with an integral timescale of about 12 days, but modes 3,4 , and 6 decay slower than mode 2 . The first seven modes decay more slowly than 6 days, and all remaining modes decay faster.

The whole idea behind the stochastic climate model is the separation between slowly evolving low-frequency (resolved) modes and considerably faster evolving unresolved modes. The stochastic mode elimination procedure (MTV01) is rigorously valid in the limit that the ratio of the autocorrelation time scale of the slowest unresolved mode to the fastest resolved modes goes to zero. Several examples from MTV02 and MTV03 show that for ratios of up to 0.5 , and sometimes even 1.0 , this approach is still applicable. For the cases presented here this ratio is relatively large; in particular, by considering only the first two modes as resolved this ratio is 1.5 , for the first four modes as climate modes this ratio becomes 1.1, and in the case of the first seven modes 0.67. As shown below, despite these large ratios the stochastic low-order models perform reasonably well.

The third-order moment as defined by

$$
\varrho_{k}(s)=\frac{\left\langle a_{k}^{2}(t+s) a_{k}(t)\right\rangle}{\left\langle a_{k}^{2}(t)\right\rangle^{3 / 2}}
$$

measures the deviations from Gaussianity. This quantity is normalized so that $\varrho_{k}(s)=0$ for Gaussian variables. The leading EOF modes of the barotropic model 


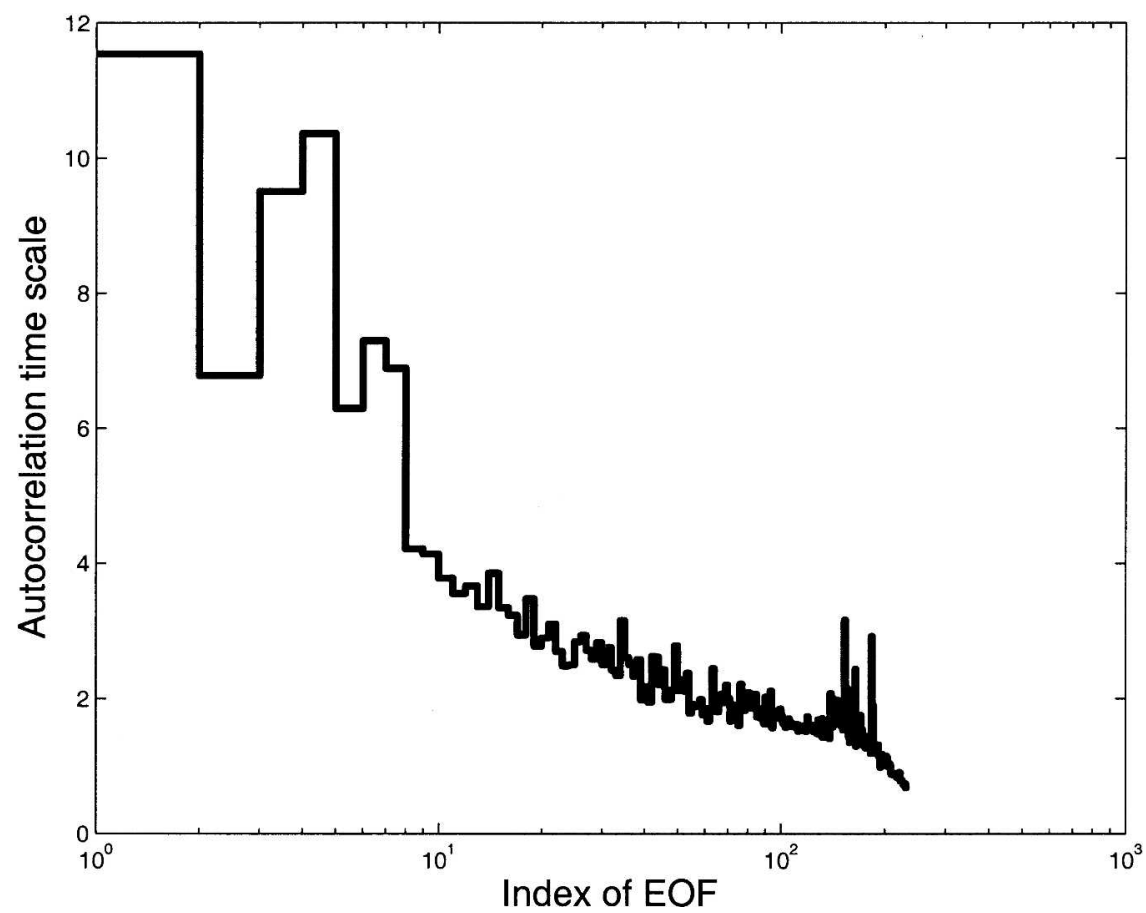

FIG. 5. Spectrum of the autocorrelation time scale.

show only modest departures from Gaussianity (Fig. 6). The first three modes show the largest departures for small lags of about 15 days, which decay quickly to zero. These modest departures from Gaussianity indicate that the EOF modes of the barotropic model are likely to be dominated by a linear Gaussian process. This is further confirmed by the one-dimensional probability density functions (PDFs) (Fig. 7). All EOF modes of the barotropic model have a quasi-Gaussian structure. To highlight this feature, we also display the corresponding theoretical Gaussian distribution together with the PDFs in Fig. 7. The barotropic model PDFs are close to their corresponding Gaussian distributions. The largest deviations from Gaussianity have EOFs 1, 3 , and 5 .

It is worth pointing out that these leading EOF patterns in the present barotropic model have a similar structure and steady-state statistics as those from a recent perpetual January simulation of the National Center for Atmospheric Research Community Climate Model (NCAR CCM0) general circulation model (Berner 2003).

\section{Systematic stochastic mode reduction}

We illustrate the ideas for stochastic climate modeling for the barotropic model in (1), which we write in the following symbolic form:

$$
\frac{\partial \psi}{\partial t}=\hat{L} \psi+B(\psi, \psi)+\hat{F}
$$

where $\psi$ denotes the streamfunction vector, $\hat{L}$ is a linear operator, $B$ is a quadratic nonlinear operator, and $\hat{F}$ denotes a constant forcing. By defining $u=\psi-\bar{\psi}$, where $\bar{\psi}$ is the time mean streamfunction, Eq. (4) becomes

$$
\begin{aligned}
\frac{\partial u}{\partial t}= & \hat{L} u+\hat{L} \bar{\psi}+B(\bar{\psi}, \bar{\psi})+B(u, \bar{\psi})+B(\bar{\psi}, u) \\
& +B(u, u)+\hat{F} .
\end{aligned}
$$

By defining a new linear operator, $L u=\hat{L} u+B(u, \bar{\psi})$ $+B(\bar{\psi}, u)$ and $F=\hat{F}+\hat{L} \bar{\psi}+B(\bar{\psi}, \bar{\psi})$, we can write this as

$$
\frac{\partial u}{\partial t}=L u+B(u, u)+F
$$

In stochastic climate modeling, the variable $u$ is decomposed into an orthogonal decomposition through the variables $\tilde{u}$ and $u^{\prime}$, which are characterized by strongly differing time scales. The variable $\tilde{u}$ denotes a low-frequency mode of the system, which evolves slowly in time compared to the $u^{\prime}$ variables. By decomposing $u=$ $\tilde{u}+u^{\prime}$ in terms of kinetic energy norm EOFs (appendix B) we can write them as

$$
u=\sum_{i=1}^{N} a_{i} \mathbf{e}_{i}=\sum_{i=1}^{R} \alpha_{i} \mathbf{e}_{i}+\sum_{j=R+1}^{N} \beta_{j} \mathbf{e}_{j},
$$

with $\tilde{u}=\sum_{i=1}^{R} \alpha_{i} \mathbf{e}_{i}$ and $u^{\prime}=\sum_{j=R+1}^{N} \beta_{j} \mathbf{e}_{j}$, where $R$ is the number of resolved modes, $a_{i}$ denote the EOF expan- 
a)

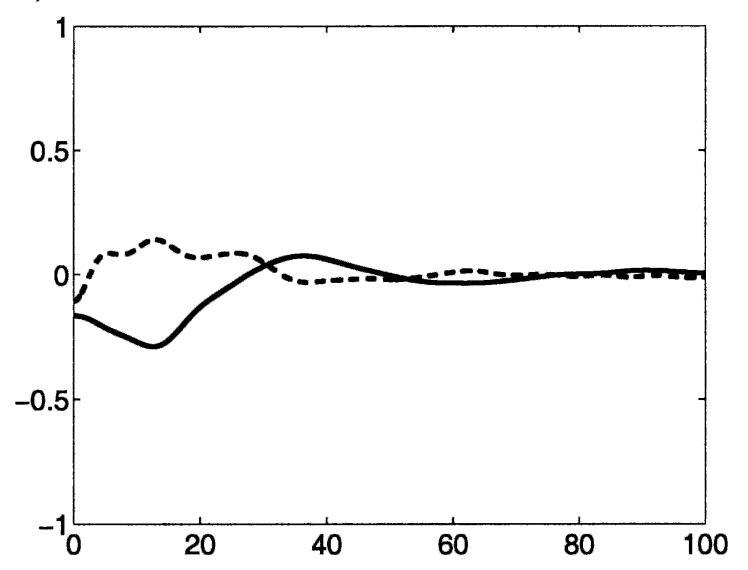

c)

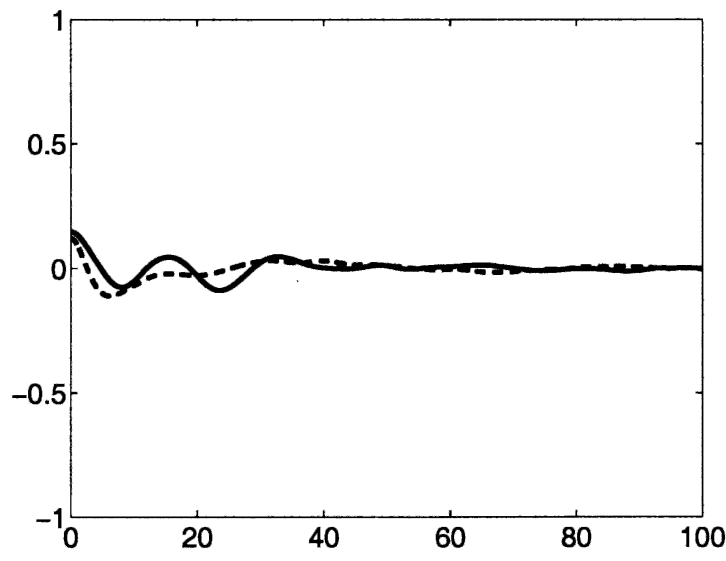

b)

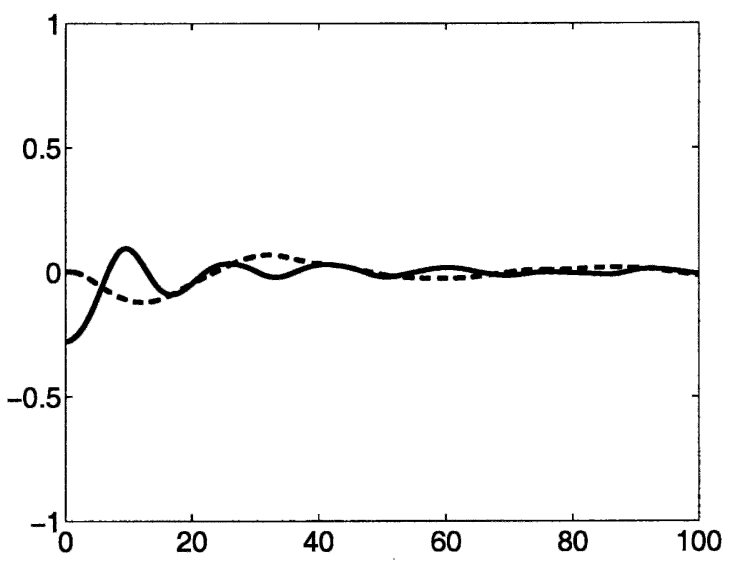

d)

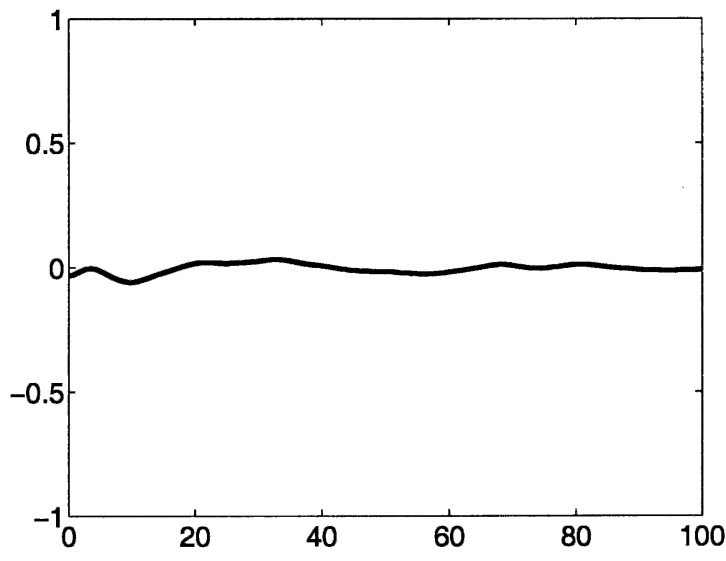

FIG. 6. Third-order moment of the amplitude of the kinetic energy norm EOFs of the barotropic model: (a) EOF1 (solid line) and EOF2 (dashed line), (b) EOF3 (solid line) and EOF4 (dashed line), (c) EOF5 (solid line) and EOF6 (dashed line), and (d) EOF7 (solid line).

sion coefficients, $\alpha_{i}\left(\beta_{j}\right)$ are the expansion coefficients of the resolved (unresolved) modes, and $N=231$ for the present application. The use of the kinetic energy norm ensures the conservation of kinetic energy by the nonlinear operator (Selten 1995; Kwasniok 1996). By properly projecting the EOF expansions, derived from the barotropic model, onto Eq. (6), we get two sets of equations for resolved $\alpha_{i}$ and unresolved $\beta_{i}$ modes:

$$
\begin{aligned}
& \dot{\alpha}_{i}(t)=\varepsilon H_{i}^{\alpha}+\sum_{j} L_{i j}^{\alpha \alpha} \alpha_{j}(t)+\frac{1}{\varepsilon} \sum_{j} L_{i j}^{\alpha \beta} \beta_{j}(t)+\sum_{j k} B_{i j k}^{\alpha \alpha \alpha} \alpha_{j}(t) \alpha_{k}(t)+\frac{2}{\varepsilon} \sum_{j k} B_{i j k}^{\alpha \alpha \beta} \alpha_{j}(t) \beta_{k}(t)+\frac{1}{\varepsilon} \sum_{j k} B_{i j k}^{\alpha \beta \beta} \beta_{j}(t) \beta_{k}(t), \\
& \dot{\beta}_{i}(t)=\varepsilon H_{i}^{\beta}+\frac{1}{\varepsilon} \sum_{j} L_{i j}^{\beta \alpha} \alpha_{j}(t)+\frac{1}{\varepsilon} \sum_{j} L_{i j}^{\beta \beta} \beta_{j}(t)+\frac{1}{\varepsilon} \sum_{j k} B_{i j k}^{\beta \alpha \alpha} \alpha_{j}(t) \alpha_{k}(t)+\frac{2}{\varepsilon} \sum_{j k} B_{i j k}^{\beta \alpha \beta} \alpha_{j}(t) \beta_{k}(t)+\frac{1}{\varepsilon^{2}} \sum_{j k} B_{i j k}^{\beta \beta \beta} \beta_{j}(t) \beta_{k}(t),
\end{aligned}
$$

where the interaction coefficients are defined in appendix $\mathrm{C}$ and the nonlinear operators have been symmetrized; that is, $B_{i j k}=B_{i k j}$ in (8) and (9). The upper indices $\alpha$ and $\beta$ indicate the respective subsets of the full operators in (6). Here $\varepsilon$ is a small positive parameter that controls the separation of time scale between slow and fast modes and measures the ratio of the correlation time of the slowest unresolved mode $u^{\prime}$ to the fastest resolved mode $\tilde{u}$. In placing the parameter in front of particular terms we tacitly assume that they evolve on a faster time scale then the terms involving the resolved modes alone. Ultimately, $\varepsilon$ is set to the 
a) EOF1

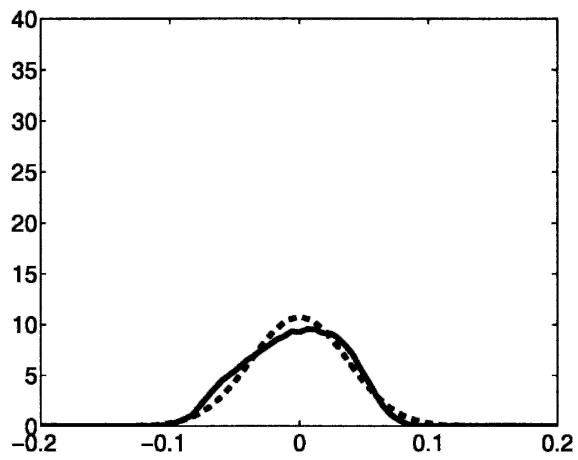

c) EOF3

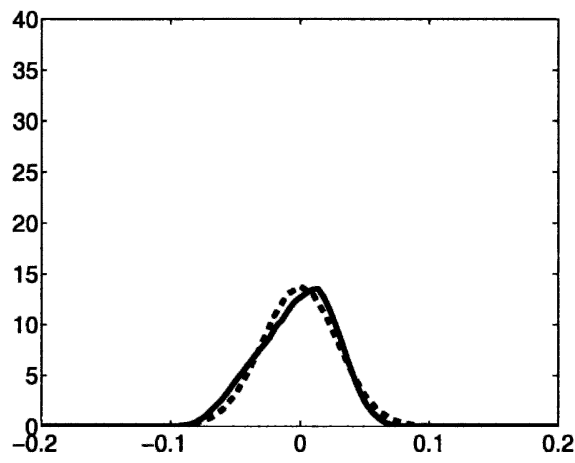

e) EOF5

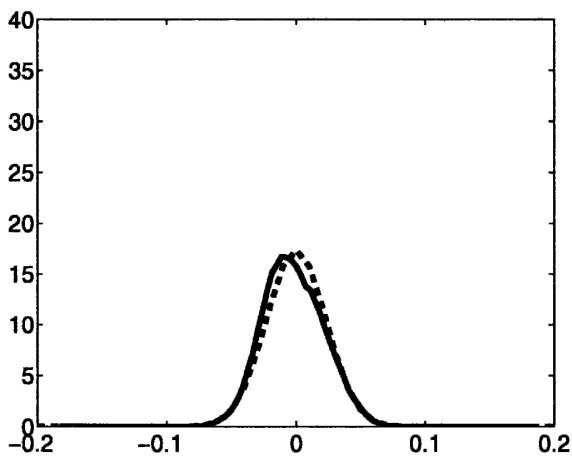

g) EOF7

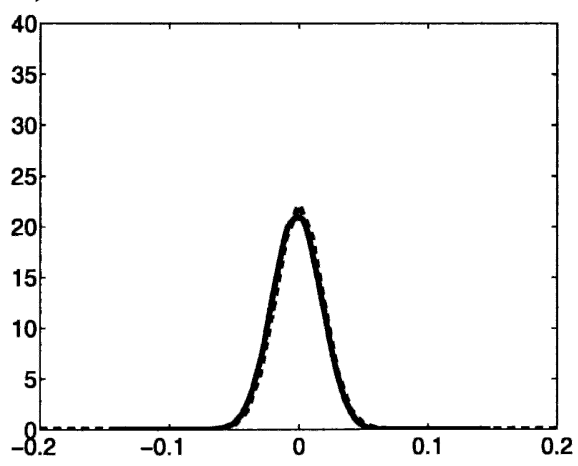

b) EOF2

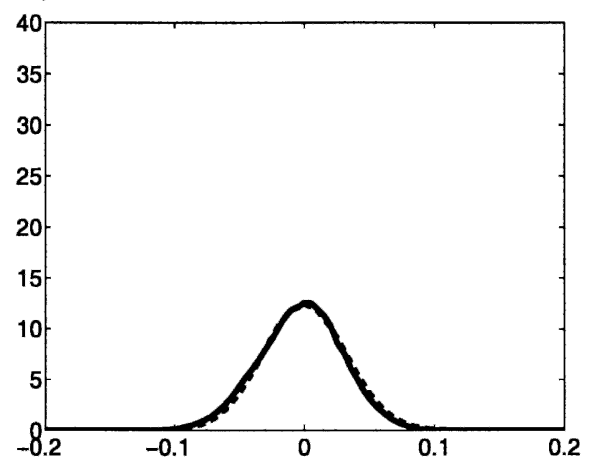

d) EOF4

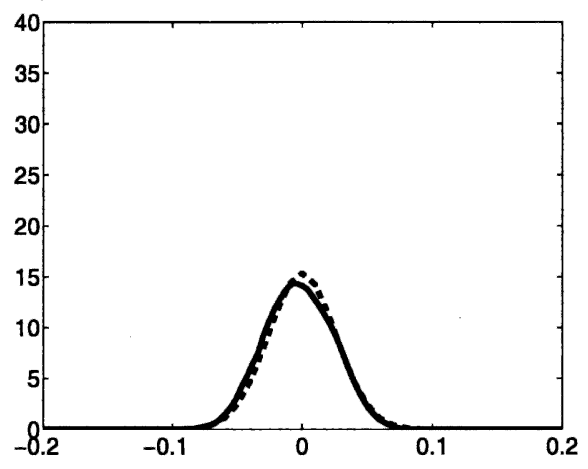

f) EOF6

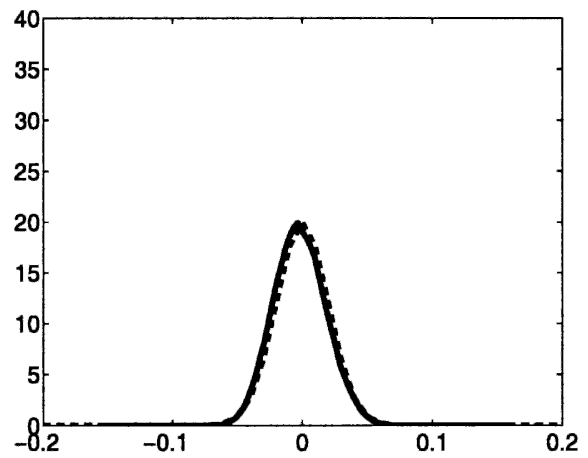

FIG. 7. PDF of the amplitude of the kinetic energy norm EOFs of the barotropic model. The solid lines are the barotropic model modes, and the dashed lines are corresponding Gaussian PDFs with the same variance as the barotropic model modes. The means are not subtracted. 
value $\varepsilon=1$ in developing all final results (MTV02, MTV03), that is, introducing $\varepsilon$ is only a technical step in order to carry out the MTV mode reduction strategy. Such a use of $\varepsilon$ has been checked on a wide variety of idealized examples where the actual value of $\varepsilon$ ranges from quite small to order 1 (MTV02, MTV03; Majda and Timofeyev 2004). In particular, here in (8) and (9) we make the following assumptions:

- The external forcings $H^{\alpha}$ and $H^{\beta}$ act on a slow time scale of order $\varepsilon$. This assumption leads to their vanishing in the effective equations.

- $L^{\alpha \alpha}$ and $B^{\alpha \alpha \alpha}$ act on a time scale of order one.

- The linear operators $L^{\alpha \beta}, L^{\beta \alpha}$, and $L^{\beta \beta}$ act at most on a faster time scale of order $\varepsilon^{-1}$.

- The self-interaction of the unresolved modes $B^{\beta \beta \beta}$ acts on the fastest time scale of order $\varepsilon^{-2}$, whereas $B^{\alpha \alpha \beta}, B^{\alpha \beta \beta}, B^{\beta \alpha \alpha}$, and $B^{\beta \alpha \beta}$ act on a time scale of order $\varepsilon^{-1}$.

Furthermore, it is assumed that the dynamical system given only by unresolved modes with interaction $B^{\beta \beta \beta}$ is ergodic and mixing, and can be represented by a stochastic process, and that all unresolved modes are quasi-Gaussian distributed (based on the model dynamics this assumption is valid). With these assumptions, the seamless MTV procedure (see appendix D) predicts the following effective stochastic equations for the resolved variables alone:

$$
\begin{aligned}
d \alpha_{i}(t)= & \sum_{j} L_{i j}^{\alpha \alpha} \alpha_{j}(t) d t+\sum_{j k} B_{i j k}^{\alpha \alpha \alpha} \alpha_{j}(t) \alpha_{k}(t) d t \\
& +\tilde{H}_{i} d t+\sum_{j} \tilde{L}_{i j} \alpha_{j}(t) d t+\sum_{j k} \tilde{B}_{i j k} \alpha_{j}(t) \alpha_{k}(t) d t \\
& +\sum_{j k l} \tilde{M}_{i j k l} \alpha_{j}(t) \alpha_{k}(t) \alpha_{l}(t) d t \\
& +\sqrt{2} \sum_{j} \sigma_{i j}^{(1)}(\alpha(t)) d W_{i}^{(1)}+\sqrt{2} \sum_{j} \sigma_{i j}^{(2)} d W_{i}^{(2)} .
\end{aligned}
$$

This stochastic differential equation system is in Itô form (Gardiner 1985). This system consists of the bare truncation (minus the bare forcing); additional new forcing; linear, quadratic, and cubic nonlinear interaction terms; as well as additive and multiplicative noises. All of these correction terms and noises are predicted by the systematic stochastic mode reduction strategy and account for the interaction between resolved and unresolved modes, as well as for the self-interaction between the unresolved modes. As described below these correction terms stem from certain physical processes. The explicit values of the coefficients in (10) are determined by the seamless MTV procedure in appendix $\mathrm{D}$ with the only inputs from the unresolved modes involving their variances and the integrated autocorrelation components (the unresolved correlation times).

To see which of these correction terms play a vital role in the integrations of the low-order stochastic model, that is, (10), we group the interaction terms between resolved and unresolved modes according to their physical origin and set a parameter $\lambda_{i}$ in front of the corresponding interaction coefficient. The interaction between the triads $B^{\alpha \beta \beta}$ and $B^{\beta \alpha \beta}$ give rise to additive noise and a linear correction term; we will name these triads additive triads and set a $\lambda_{A}$ in front of them (MTV99, MTV01, MTV02, MTV03). The second type of triad interaction is between $B^{\alpha \alpha \beta}$ and $B^{\beta \alpha \alpha}$. These interactions create multiplicative noises and cubic nonlinear correction terms (MTV99, MTV01, MTV02, MTV03); we will call them multiplicative triads in the following and indicate them by a $\lambda_{M}$. The linear coupling between the resolved and unresolved modes $L^{\alpha \beta}$ and $L^{\beta \alpha}$ give rise to additive noise and a linear correction term (MTV01), which are called the augmented linearity here, indicated by a $\lambda_{L}$. We set a $\lambda_{F}$ in front of the last remaining interaction term $L^{\beta \beta}$, the linear coupling of the unresolved modes. The quadratic nonlinear corrections, a forcing term, and a further multiplicative noise contribution are caused by the interaction between the linear coupling terms and the multiplicative triads. Another forcing correction term comes from the interaction between additive triads and the linear coupling of the fast modes.

After regrouping the terms the stochastic climate model from (10) can be written according to these physical processes as

$$
\begin{aligned}
d \alpha_{i}(t)= & \sum_{j} L_{i j}^{\alpha \alpha} \alpha_{j}(t) d t+\sum_{j k} B_{i j k}^{\alpha \alpha \alpha} \alpha_{j}(t) \alpha_{k}(t) d t \\
& +\lambda_{A}^{2} \sum_{j} \tilde{L}_{i j}^{(2)} \alpha_{j}(t) d t+\lambda_{A} \sqrt{2} \sum_{j} \sigma_{i j}^{(2)} d W_{j}^{(2)} \\
& +\lambda_{M}^{2}\left(\sum_{j} \tilde{L}_{i j}^{(3)} \alpha_{j}(t) d t+\sum_{j k l} \tilde{M}_{i j k l} \alpha_{j}(t) \alpha_{k}(t) \alpha_{l}(t) d t\right) \\
& +\lambda_{L}^{2}\left(\sum_{j} \tilde{L}_{i j}^{(1)} \alpha_{j}(t) d t\right) \\
& +\lambda_{M} \lambda_{L}\left(\tilde{H}_{j}^{(1)} d t+\sum_{j k} \tilde{B}_{i j k} \alpha_{j}(t) \alpha_{k}(t) d t\right) \\
& +\lambda_{A} \lambda_{F} \tilde{H}_{j}^{(2)} d t \\
& +\sqrt{2} \sum_{j} \sigma_{i j}^{(1)}(\alpha(t)) d W_{j}^{(1)},
\end{aligned}
$$

where the nonlinear noise matrix $\boldsymbol{\sigma}^{(1)}$ satisfies

$$
\begin{aligned}
\lambda_{L}^{2} Q_{i j}^{(1)}+\lambda_{L} \lambda_{M} \sum_{k} U_{i j k} \alpha_{k}(t)+\lambda_{M}^{2} \sum_{k l} V_{i j k l} \alpha_{k}(t) \alpha_{l}(t) \\
=\sum_{k} \sigma_{i k}^{(1)}(\alpha(t)) \sigma_{j k}^{(1)}(\alpha(t)) .
\end{aligned}
$$

It is guaranteed (MTV01) that the operator on the lefthand side of (12) is always positive definite, ensuring the existence of the nonlinear noise matrix on the righthand side. All coefficients are defined explicitly in appendix D. 
The first line in Eq. (11) is the bare truncation (minus bare forcing), the second line denotes the additive triad contributions. The third line indicates the multiplicative triad contributions, the fourth line is the augmented linearity, the fifth line denotes the interaction between multiplicative triads and the linear coupling, and the sixth line comes from the interaction between additive triads and the linear coupling of the fast modes. The last line and (12) determine a multiplicative noise operator, whose contributions stem from the linear coupling, the multiplicative triads, and the interaction between multiplicative triads and the linear coupling. By considering only contributions from the linear coupling, in other words setting $\lambda_{M}=0$ in (12), this multiplicative noise operator reduces to an additive noise operator.

For integrating this stochastic differential equation a split step numerical procedure is utilized with a fourthorder Runge-Kutta scheme for the deterministic part and an Euler-forward scheme for the stochastic part. A time step size of 0.01 days has been used for all integrations. Statistics of the stochastic model are calculated by time averaging an individual solution integrated over a long time of the order of $10^{6}$ days.

\section{Stochastic climate model results}

\section{a. Bare truncation}

First, we test the performance of the low-order model without any closure to account for the neglected interactions with the unresolved EOFs, the so-called bare truncation (this contains the bare forcing). This is done to see how well the bare truncation dynamics capture the projected dynamics of the EOFs of the original barotropic model. To analyze the flow characteristics of these low-order systems we integrate the model starting from 10000 normally distributed initial conditions for 1000 days and save only the last model state. Integrations go to a fixed point for low-order models consisting of only two or three resolved modes. The fixed point is always the same for the respective loworder model. Low-order models (those with 4 to 7 modes) go into a stable periodic orbit (not shown). The period orbit is the same for every realization. This indicates that the bare truncation does not capture the dynamics of the leading EOF modes of the barotropic model very well.

Long integrations for single realizations show that the bare truncation low-order models have large means (the barotropic model mode means are zero) and too much variance. The autocorrelation function of the 7-mode system is dominated by a stable oscillation (Fig. 14). The corresponding PDFs show a double peak structure and also too much variance (Fig. 15). These results emphasize the importance of properly accounting for the neglected unresolved interactions. Climate drifts and too-large variances of truncated EOF models have also been reported by Selten (1995), Achatz and
Branstator (1999), and for PIP models (Kwasniok 1996). These studies add linear damping to their models in order to eliminate the climate drift and to reduce the variances.

\section{b. Stochastic climate model with only diagonal correlations for unresolved modes}

Now we apply the systematic stochastic mode reduction strategy that properly accounts for the neglected interactions with the unresolved modes. In appendix D we develop a general framework for stochastic mode reduction, which was summarized in the last section. First we will present results from a simplified framework, which requires the least input of information from the unresolved modes where we assume that all cross correlations are zero between the unresolved modes. This means that we set $\mathcal{B}_{i j}(t)=\mathcal{B}_{i}(t) \delta_{i j}$ in Eqs. (D16)-(D31). Results for the general strategy will be presented in the next section.

First we describe the results for the 4-mode reduced stochastic system. The means of the individual modes are small; therefore, the climatological streamfunction is very close to the barotropic model mean (not shown). The amplitude is well captured and the pattern correlation between both model means is 0.99 (Table 1). Figure 8 shows the horizontal distribution of the standard deviation of the streamfunction and the climatological transient eddy forcing $-\nabla^{-2} \nabla \cdot\left(\overline{\mathbf{u}^{\prime} \zeta^{\prime}}\right)$. To allow for a fair comparison, the corresponding barotropic model standard deviation and transient eddy forcing have been calculated from the first four EOFs only. The stochastic model reproduces the key features of the standard deviation quite well. Only the amplitude is underestimated by a factor of about 2. The pattern correlation between both is 0.98 . Also the key features of the climatological transient eddy forcing are reproduced fairly well, although the amplitude is again underestimated. The pattern correlation for the transient eddy forcing is 0.91 . Figure 9 shows the autocorrelation functions of the four leading modes in the reduced model. It captures roughly most of the oscillations of modes 2 and 4 . The stochastic climate model does not reproduce the oscillations of modes 1 and 3 . The stochastic climate model reproduces reasonably well the low-frequency envelop of the autocorrelation function of the original EOFs of the barotropic model,

TABLE 1. Pattern correlation between barotropic model and stochastic model for various truncations. To allow for a fair comparison, the corresponding barotropic model fields have been calculated with the same number of EOFs as for the stochastic model.

\begin{tabular}{cccc}
\hline $\begin{array}{c}\text { Number } \\
\text { of modes }\end{array}$ & Mean & Std dev & $\begin{array}{c}\text { Transient } \\
\text { eddy forcing }\end{array}$ \\
\hline 2 & 0.99 & 0.98 & 0.86 \\
4 & 0.99 & 0.98 & 0.91 \\
7 & 0.99 & 0.99 & 0.97 \\
\hline
\end{tabular}


a)

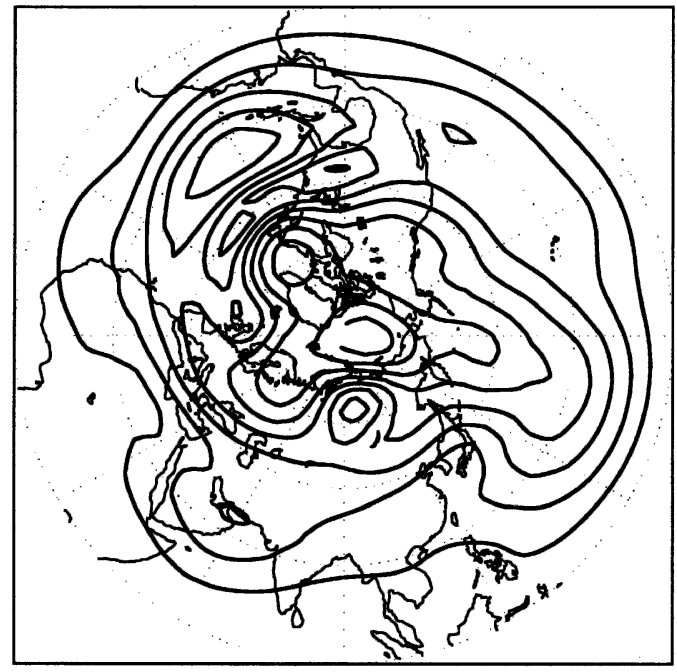

c)

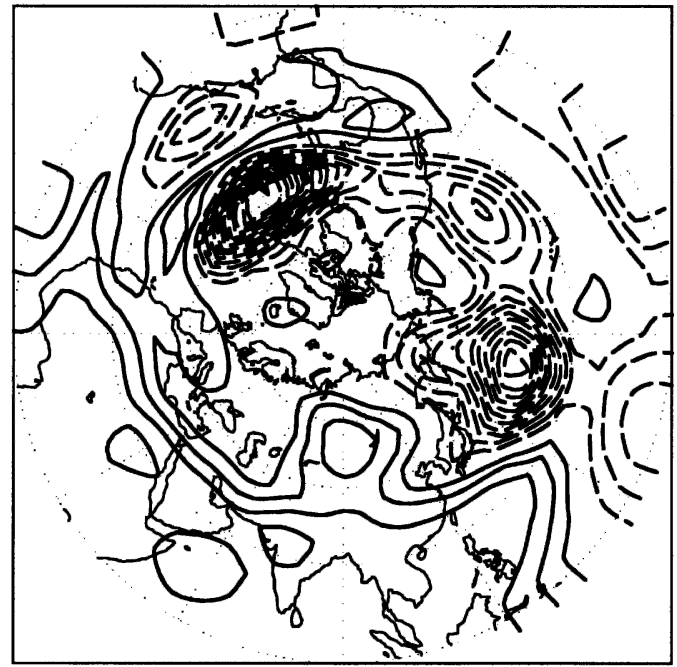

b)

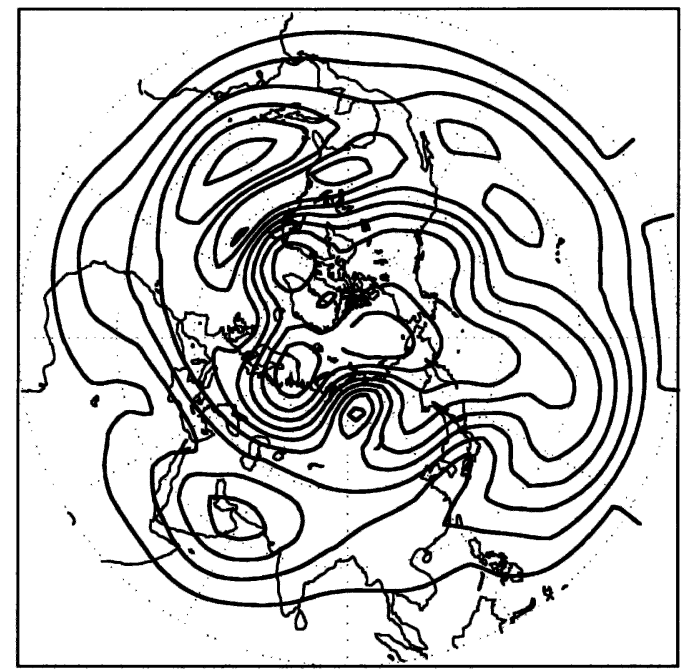

d)

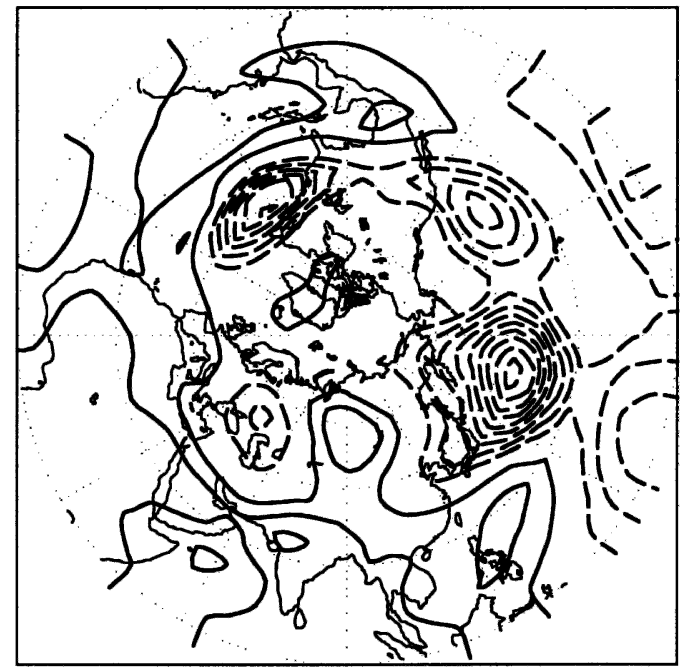

FIG. 8. Horizontal distribution of streamfunction standard deviation: (a) barotropic model (contour interval is $5 \times 10^{5} \mathrm{~m}^{2} \mathrm{~s}^{-1}$ ), and (b) 4-mode stochastic model (contour interval is $2 \times 10^{5} \mathrm{~m}^{2} \mathrm{~s}^{-1}$ ). Transient eddy forcing: (c) barotropic model (contour interval is $5 \mathrm{~m}^{2} \mathrm{~s}^{-2}$ ), and (c) 4-mode stochastic model (contour interval is $2 \mathrm{~m}^{2} \mathrm{~s}^{-2}$ ). For this comparison the barotropic model streamfunction has been reconstructed from the first four EOFs.

as can be seen in a semilogarithmic plot of the absolute value of the autocorrelation function (Fig. 10). The lowfrequency envelop of the barotropic model is well captured by modes 1,3 , and 4 and slightly overestimated for mode 2. The largest discrepancies occur for lags larger than 60 days. At these large lags the autocorrelation functions have already decayed to small values; therefore, these differences are more likely due to numerical errors than any systematic differences of the models. The third-order moment shows deviations from Gaussianity that are roughly of the same magnitude as for the modes of the barotropic model (not shown). The PDFs (Fig. 11) have a nearly Gaussian structure. They also show that the stochastic climate model underestimates the variances by a factor of about 1.5 to 2 . In comparison with the bare truncation integration, the predicted correction terms and noises improve the results considerably. It has to be noted that, by assuming that the resolved forcing $H_{i}^{\alpha}$ in (9) acts on a time scale of the order of 1 instead of $\varepsilon$ (as assumed above), the stochastic model experiences a climate drift. These means are still considerably smaller than the means of the bare truncation integrations. The assumption that the resolved forcing $H_{i}^{\alpha}$ acts on a time scale of the order of 1 has only a negligible effect on the autocorrelation func- 
a) Mode 1

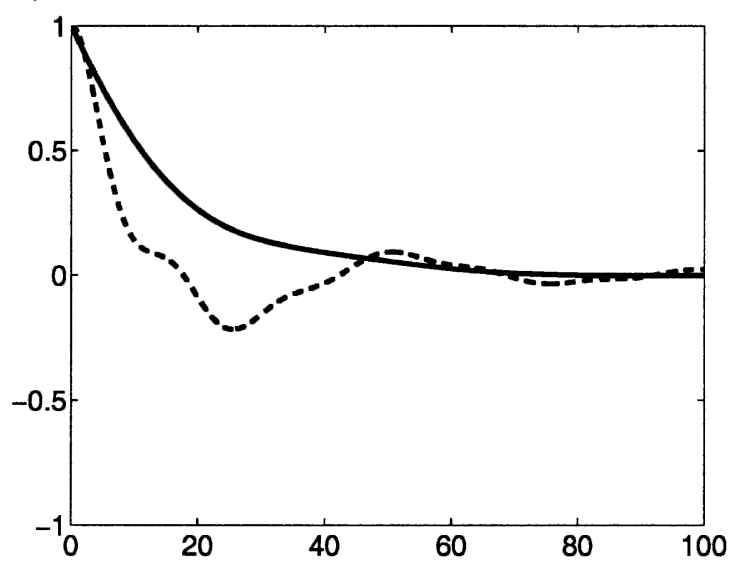

c) Mode 3

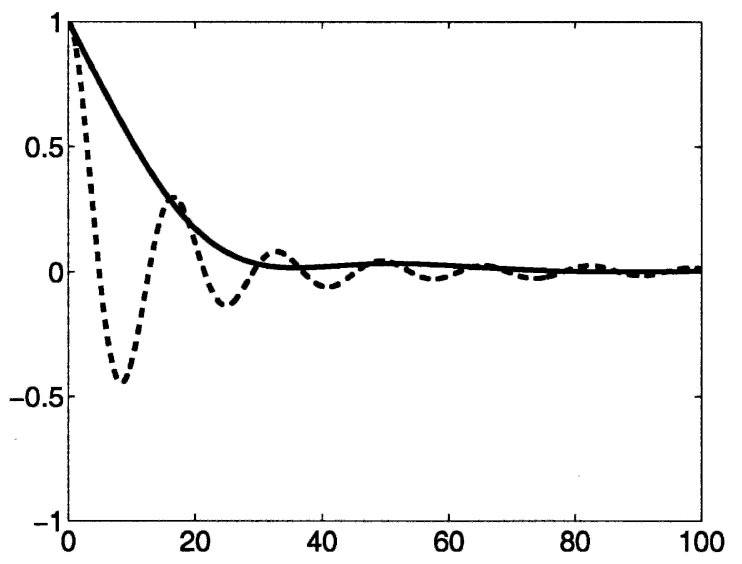

b) Mode 2

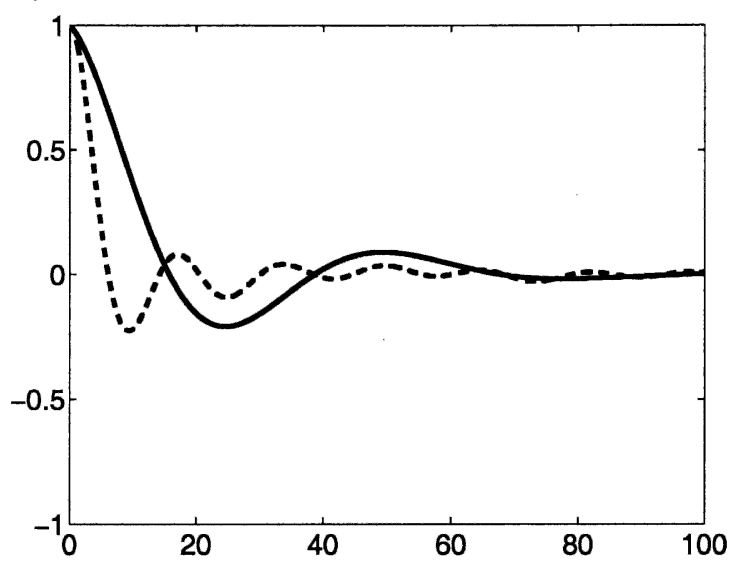

d) Mode 4

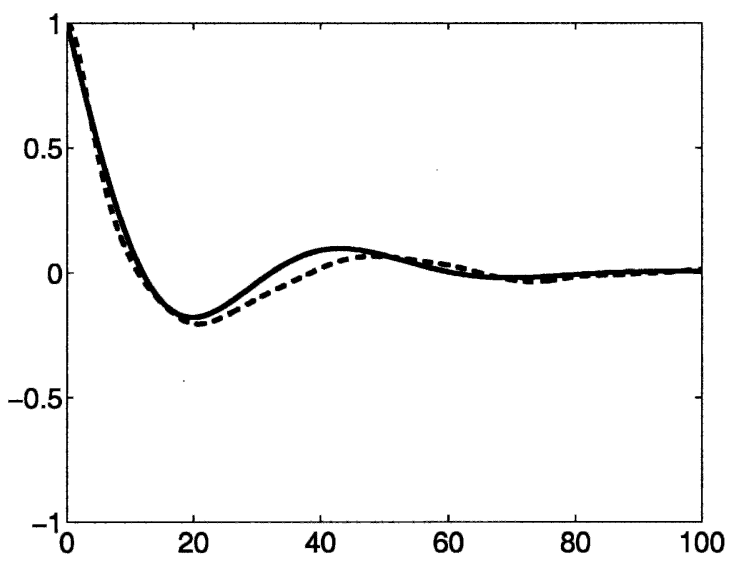

FIG. 9. Autocorrelation function for the 4-mode system; solid line is stochastic model, and dashed line is barotropic model. Only diagonal correlations are used.

tions, the third-order moments, and the variances (not shown).

Also for the 2-mode system with only diagonal correlations the geographical distribution of the means, standard deviations, and transient eddy forcing agree reasonably well with the barotropic model (Table 1). Figure 12 shows the autocorrelation function for the 2 -mode system. Mode 1 has not changed in comparison with the 4-mode system (Fig. 9a) while mode 2 has lost its oscillation; but again the 2-mode low-order model captures roughly the low-frequency envelop of the barotropic model modes reasonably well (Figs. 12c,d). Also in this case we get quasi-Gaussian PDFs, and the stochastic climate model underestimates the variances by a factor of 1.5 to 2 (Fig. 13), but less than for the 4-mode system. The third-order moments show deviations from Gaussianity that are of the right strength (not shown). Recall that this 2-mode stochastic model is a systematically derived numerical model for the two leading principal teleconnection patterns with $\mathrm{AO}$ and PNA behavior.

By increasing the number of resolved modes to 7 , the geographical distribution of the means, standard deviations, and transient eddy forcing are reasonably well reproduced again (Table 1 ). The pattern correlation for the mean streamfunction and the standard deviation is still high and the pattern correlation for the transient eddy forcing has improved to 0.97 . Only the amplitude of the standard deviation and transient eddy forcing are underestimated (not shown). The autocorrelation functions of the first three modes are further improved (Fig. 14). Modes 1 and 2 follow more closely the barotropic model modes and mode 1 also exhibits a dip around lag 25 days. Mode 3 now also shows an oscillation, which is much slower than the corresponding barotropic model mode oscillation (Fig. 14c). The full autocorrelation function is reproduced well for modes 6 and 7. Overall, the autocorrelation functions of the stochastic climate model captures the low-frequency envelope of the barotropic model modes. The corresponding PDFs are nearly Gaussian (Fig. 15). The stochastic climate model underestimates the variances by a factor of about 2 to 3 for the first 5 modes and has the right variances for mode 6 and 7. Also this stochastic climate model has 
a) Mode 1

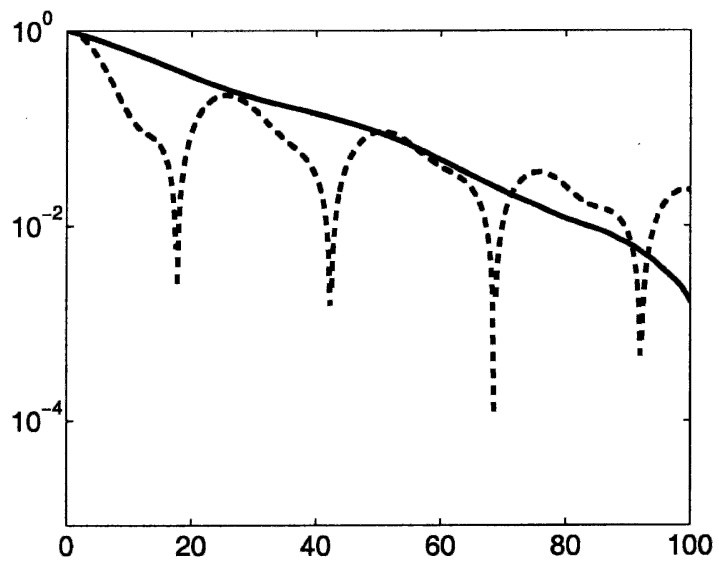

c) Mode 3

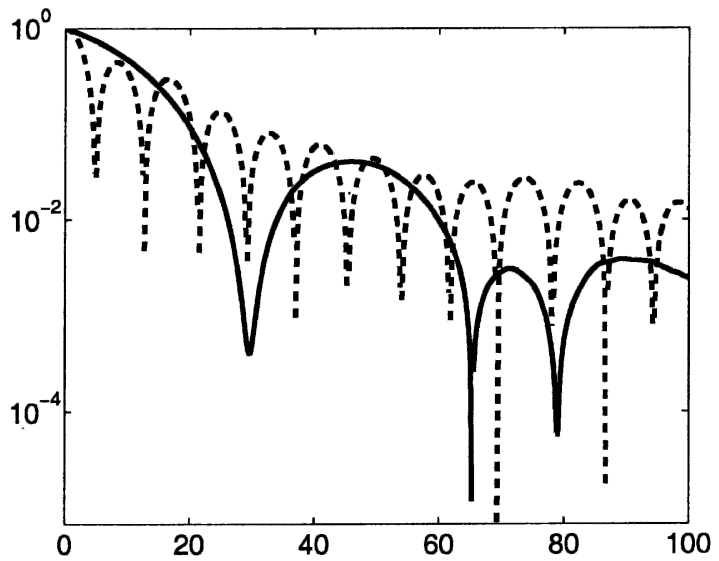

b) Mode 2

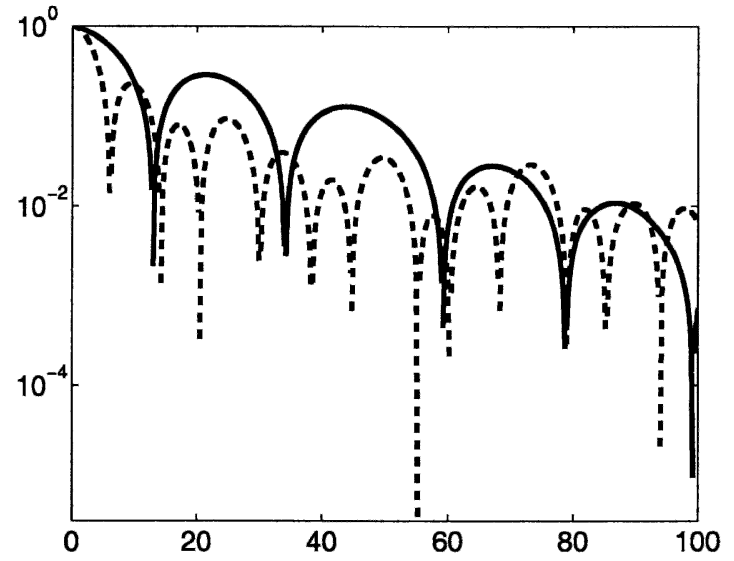

d) Mode 4

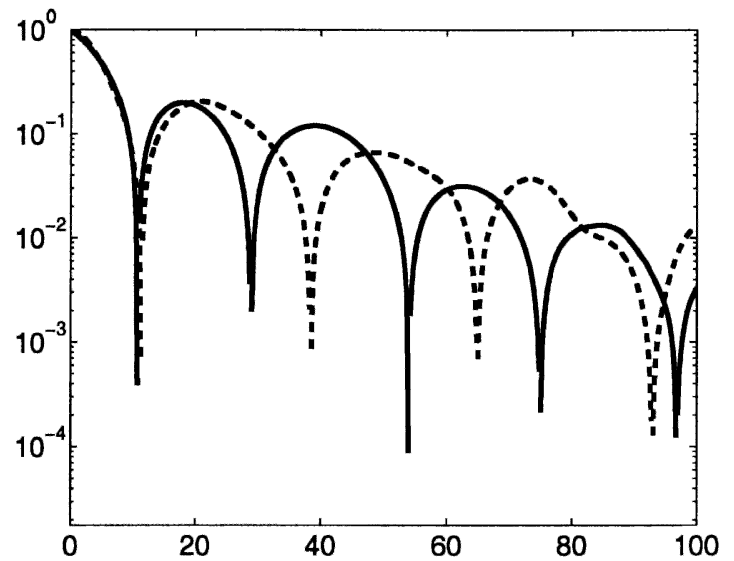

FIG. 10. Autocorrelation function for the 4-mode system using a semilogarithmic axis. Solid line is stochastic model, and dashed line is barotropic model. Only diagonal correlations are used.

vanishing means and the deviations from Gaussianity are of the right order (not shown).

The low-order stochastic models reproduce the geographical distribution of the means, standard deviations, and transient eddy forcing well. Furthermore, they capture reasonably well the low-frequency oscillations of the modes of the barotropic model but have some difficulties simulating the high-frequency oscillations. All low-order stochastic models presented above have modes that are more persistent than the corresponding barotropic model modes. They all underestimate the variances but possess self-consistent vanishing means. By comparing these results with the corresponding integrations of the bare truncation, the predicted correction terms and noises reduce the means and variances considerably. Now they have roughly the right sizes and are no longer orders of magnitude off as for the bare truncation integrations. Another property is that the variances are roughly equally distributed between the various modes; the variance does not decrease as rapidly with increasing mode number as for the barotropic model modes. However, by considering that we developed very low-order models with as little as only two resolved modes, the stochastic climate model reproduces reasonably well the key statistical features of the leading EOF modes for the barotropic model. Furthermore, this was achieved without ad hoc additions of dissipation and white noise forcing but such terms were produced automatically from the systematic procedure itself. As indicated by the autocorrelation time spectrum in Fig. 5, as in many closure procedures, one should not expect convergence of the stochastic mode reduction procedure as the number of resolved modes $R$ increases since the ratio of correlation times remains about 1 for large $R$.

\section{c. Stochastic climate model with full cross correlations for unresolved modes}

Now we present results from the general stochastic mode reduction strategy as developed in appendix D 
a) Mode 1

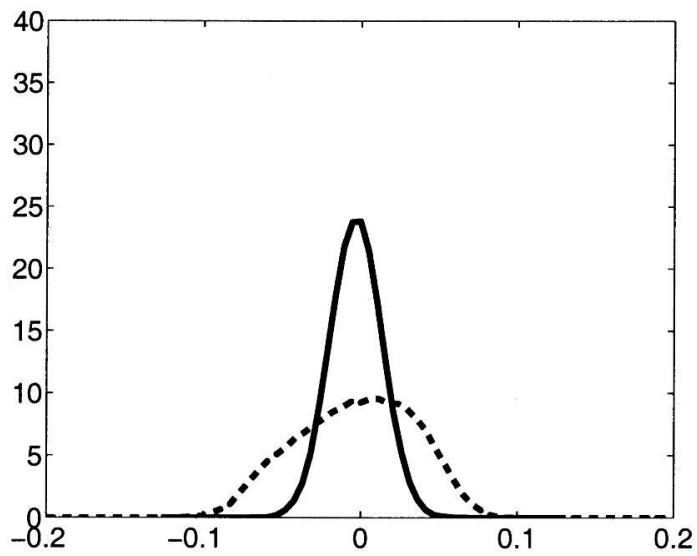

c) Mode 3

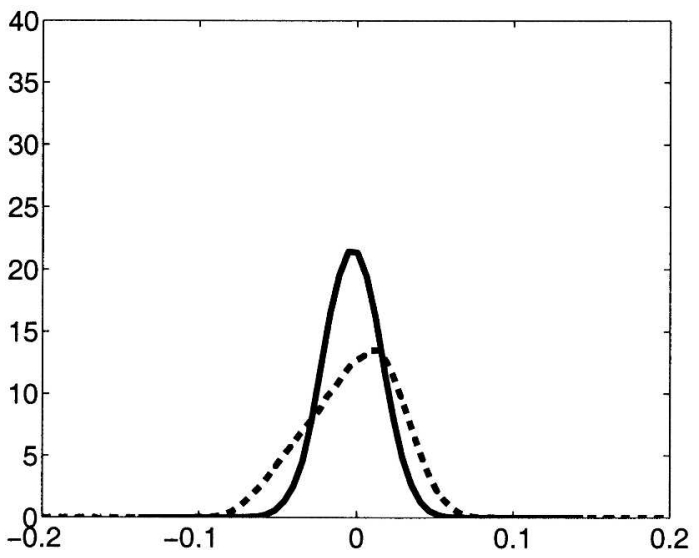

b) Mode 2

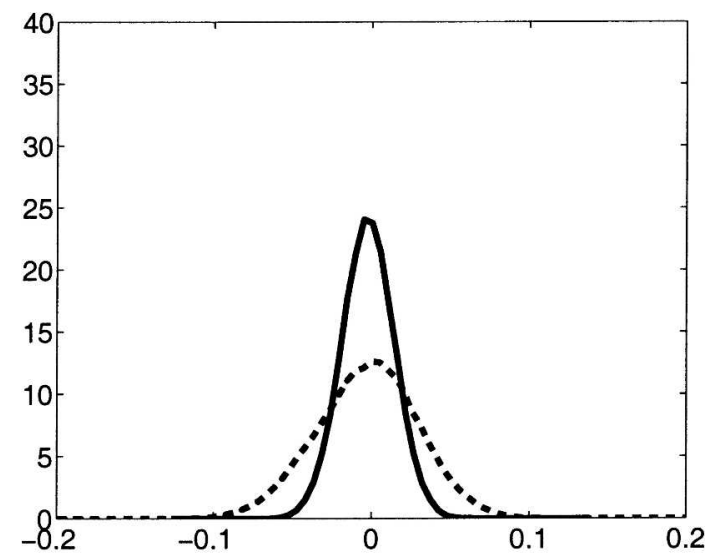

d) Mode 4

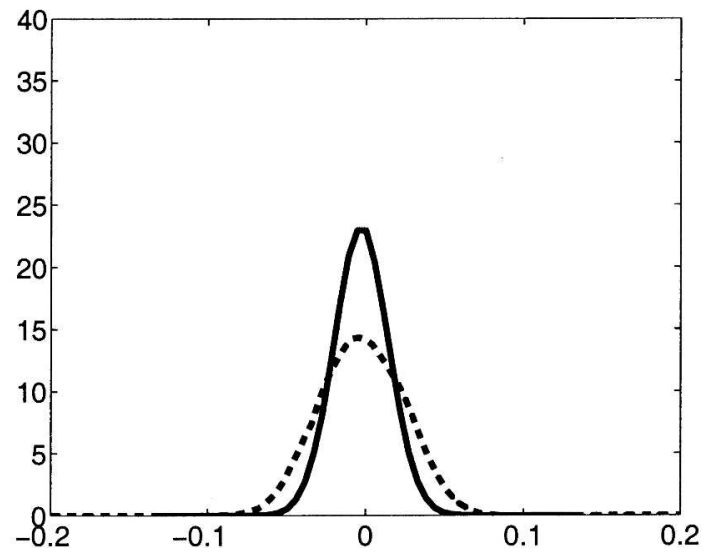

FIG. 11. PDF for the 4-mode system. Solid line is stochastic model, and dashed line is barotropic model. The means are not subtracted.

with the full matrix of unresolved cross-correlation times. We will focus on the 4-mode system; the same conclusions apply to the 2- and 7-mode systems.

As for the simplified diagonal strategy discussed earlier the low-order stochastic model captures the lowfrequency envelope of the autocorrelation function of the barotropic model modes (Fig. 16). Now mode 1 decays faster and the oscillation of mode 2 is weaker and is a better fit for the low-frequency envelope of the barotropic model mode, and mode 4 is again very close to the barotropic model mode (Fig. 9). Including the cross correlations leads to only minor changes of the variances and means (not shown). This indicates that the cross correlations of the unresolved modes have an effect on the resolved modes. They lead to improvements for certain modes, but do not affect the means and variances.

\section{d. Budget analysis}

The above sections show that the systematic loworder stochastic climate model is performing reason- ably well. This raises the question regarding which of the various correction terms in (10) are important. We will now investigate this question. In Eq. (11) the correction terms are decomposed corresponding to their physical origin. A close investigation of the various correction terms and noises reveals that the augmented linearity, $\lambda_{L}$ together with its associated additive noise term, produce the largest tendencies. The tendencies coming from the remaining correction terms and noises are at least an order of magnitude smaller during the integration of the model. By including only the bare truncation terms (minus bare forcing), the augmented linearity and its associated noise term, we are able to reproduce the results of the full stochastic climate model integrations as described above (not shown). This amounts to setting $\lambda_{L}$ to 1 and all other $\lambda_{i}$ to zero in (10).

Now the question arises how well a purely linear model with additive noise could perform. This is an interesting question since most of the previous studies on stochastic climate modeling use a linear model with 
a) Mode 1

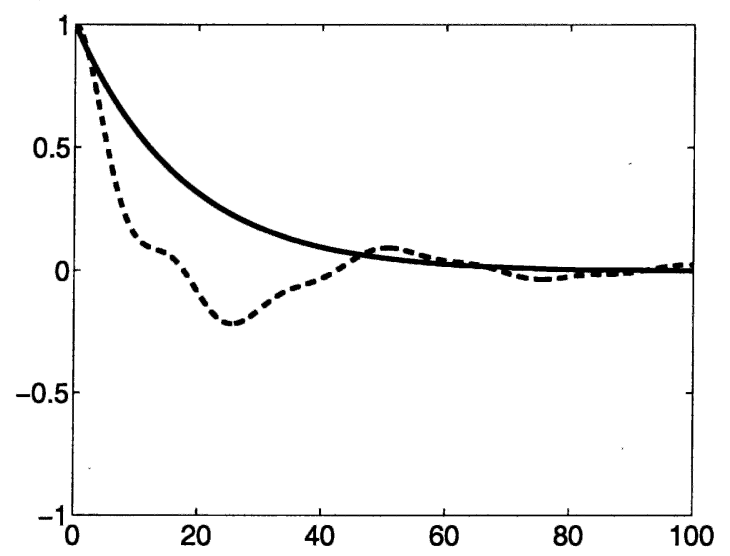

c) Mode 1

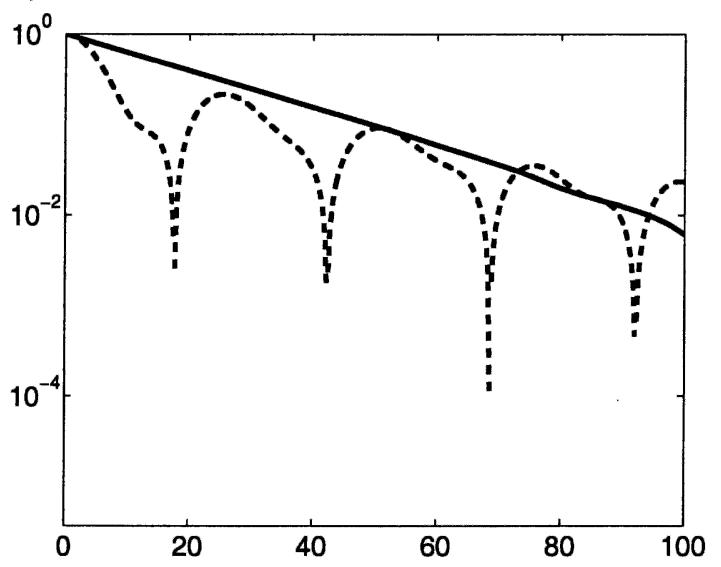

b) Mode 2

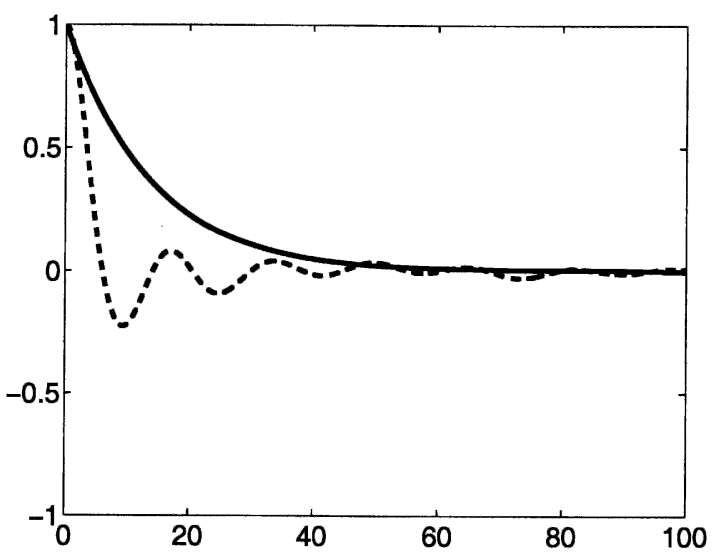

d) Mode 2

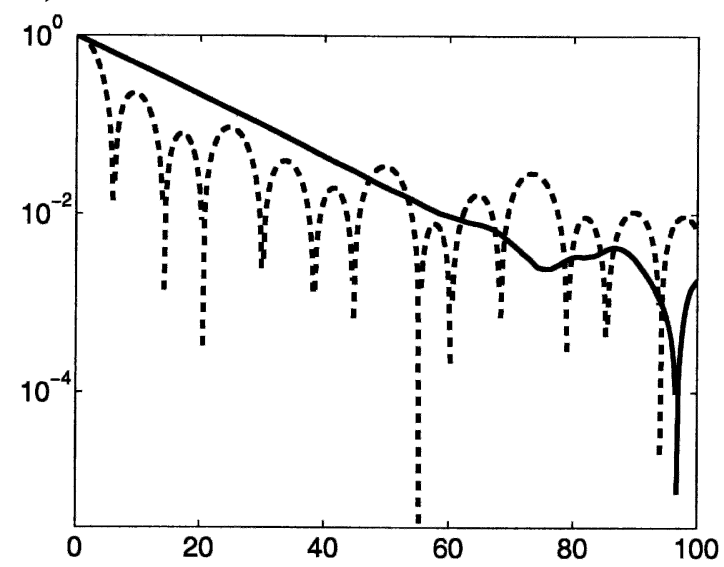

FIG. 12. Autocorrelation function for the 2-mode system. Solid line is stochastic model, and dashed line is barotropic model. Only diagonal correlations are used.

additive noise. To test this hypothesis we use only the linear part of the bare truncation (minus bare forcing), the augmented linearity correction terms, and its associated additive noise term to integrate the low-order stochastic climate model. This integration shows that such a linear stochastic model reproduces the full stochastic climate model results nearly perfectly (Fig. 17). There are only some minor differences in the autocorrelation functions of mode 3 and 4 (cf. Figs. 16 and 17). The differences in the variances and means are only marginal between the full stochastic model and the linear stochastic model (not shown).

It is important to point out that the linear correction terms and the additive noise stem from the linear coupling between the resolved and unresolved modes as shown in (11). In most previous studies about stochastic climate modeling it is argued that the added additive noise accounts for the neglected nonlinear interactions and the forcing (Newman et al. 1997; Winkler et al. 2001). In terms of the present context these studies only consider the additive triads, which are leading to a linear correction term and additive noise. These correc- tion terms have a negligible role in the present study. Also the corrections terms stemming from multiplicative triads, which lead to the other nonlinear correction terms, are negligible in this study.

\section{Summary and discussion}

This study applies a systematic strategy for stochastic mode reduction to a realistic barotropic model climate. The barotropic model climate has realistic versions of the AO and PNA teleconnection patterns as the leading EOFs. The systematic strategy requires first the identification of slowly evolving climate modes and fast evolving nonclimate modes. The low-order stochastic climate model consists of the climate modes as resolved modes and the stochastic mode reduction procedure predicts all forcing, linear, quadratic, and cubic correction terms as well as additive and multiplicative noises; these correction terms and noises account for the interaction of the resolved climate modes with the neglected nonclimate modes and for the self-interaction of the 
a) Mode 1

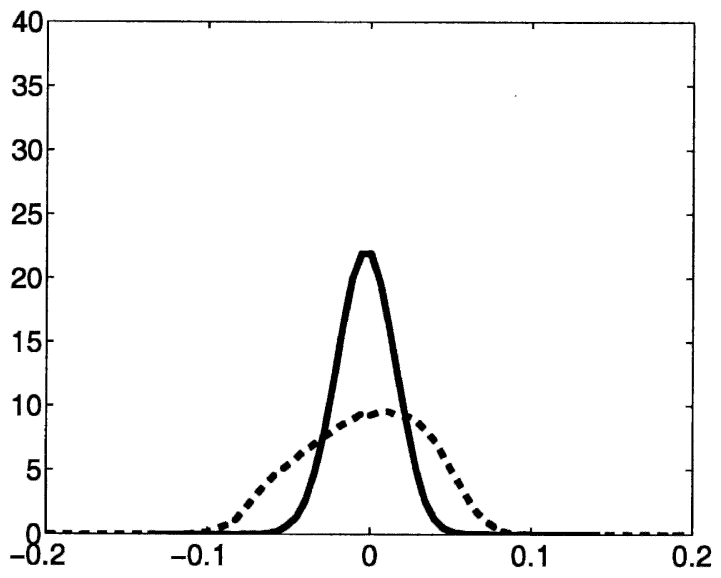

b) Mode 2

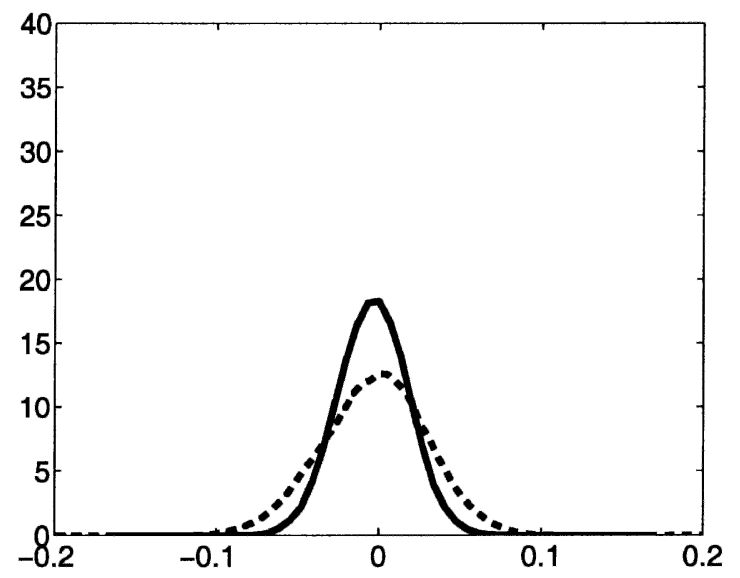

FIG. 13. PDF for the 2-mode system. Solid line is stochastic model, and dashed line is barotropic model. The means are not subtracted.

nonclimate modes. The stochastic mode reduction strategy presented here is a generalized framework of that presented in MTV99, MTV01, MTV02, and MTV03, which is more suitable for implementation in other complex geophysical systems with minimal fitting of the unresolved modes.

The stochastic mode elimination procedure is justified rigorously for $\varepsilon=1$, where $\varepsilon$ measures the ratio of correlation times of unresolved modes to the resolved ones. MTV02, MTV03 show that the strategy applies for values of $\varepsilon$ as large as 0.5 and even for 1 in many situations. For the cases presented here $\varepsilon$ is relatively large; in particular for the 2-mode low-order model this ratio is 1.5 , for the 4 -mode model 1.1, and for the 7-mode model 0.67. Despite these large ratios the loworder models perform reasonably well.

The low-order stochastic models reproduce the geographical distribution of the climatological means, standard deviation, and transient eddy forcing reasonably well. On the other hand, they underestimate the amplitudes of the standard deviation and of the transient eddy forcing by a factor of about 2 . Low-order models with four and seven modes capture the autocorrelation functions fairly well. These results provide evidence for effective stochastic dynamics despite the minimal timescale separation between resolved and unresolved modes.

Kwasniok (2004) points out that for deriving loworder models based on PIPs for a similar barotropic model climate, at least 15 PIP modes are needed to reproduce the means and variances reasonably well. To capture the temporal characteristics, the autocorrelation function and the PDFs, at least 40 PIPs are needed. A similar EOF model needs 100 EOFs to model the system as well as with 40 PIPs. In a baroclinic model, Achatz and Branstator (1999) report that their EOF model needs between 30 and 70 EOFs to simulate the climate mean state and transient eddy fluxes. The pre- sented systematic stochastic mode reduction strategy leads to much lower-order models with qualitatively comparable results.

A budget analysis reveals that the minimal stochastic model dynamics consists of the linear part of the bare truncation and the augmented linearity. The augmented linearity accounts for the linear coupling between the resolved and unresolved modes. It consists of a linear correction term and additive noise. This minimal linear stochastic model reproduces the statistics of the full low-order stochastic model within minor discrepancies. This linear stochastic model is different from previous proposed linear stochastic models (Newman et al. 1997; Whitaker and Sardeshmukh 1998; Winkler et al. 2001). These studies approximate the nonlinear part of the equations by a linear operator and additive noise. This noise is typically white in time but may be spatially correlated. In other words, they truncate the dynamics on both the resolved and unresolved modes, and add ad hoc damping, necessary to stabilize the model (Whitaker and Sardeshmukh 1998). The approach presented here truncates the dynamics only on the unresolved modes and predicts all necessary corrections and noises; therefore, it also predicts the necessary damping. Furthermore, the systematic approach does not assume anything a priori about the dominant dynamics, such as linearity. It is rather a result of the stochastic mode reduction strategy that for the model climate considered here midlatitude low-frequency variability can be modeled by a linear low-order stochastic model. Further evidence for the dominance of linear dynamics in reduced barotropic models has been provided by Kwasniok (2004). In his empirical low-order model based on PIPs, the linear interaction terms dominated over the nonlinear interaction terms.

Kwasniok shows that reduced PIP models are superior to low-order models based on EOFs. On the other 
a) Mode 1

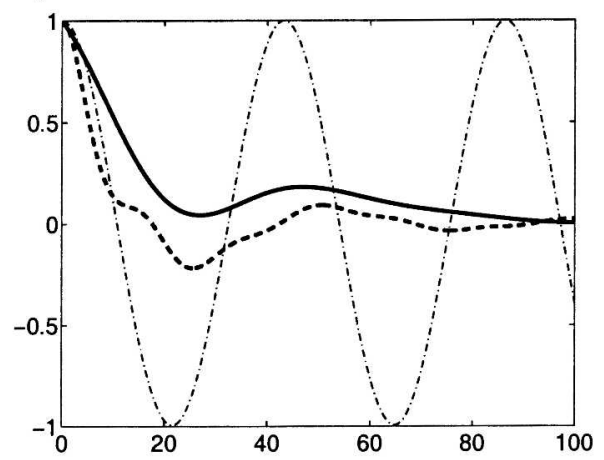

c) Mode 3

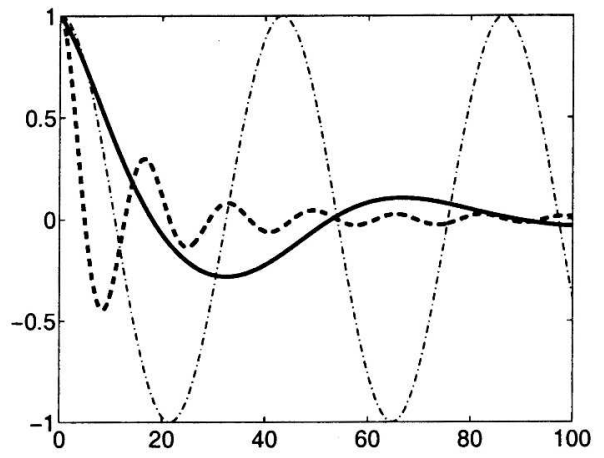

e) Mode 5

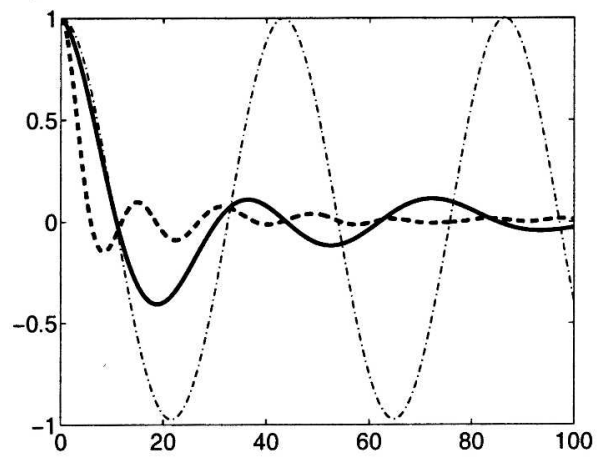

g) Mode 7

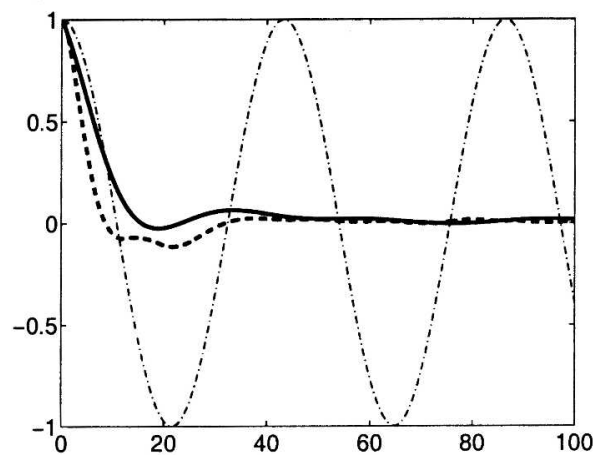

b) Mode 2

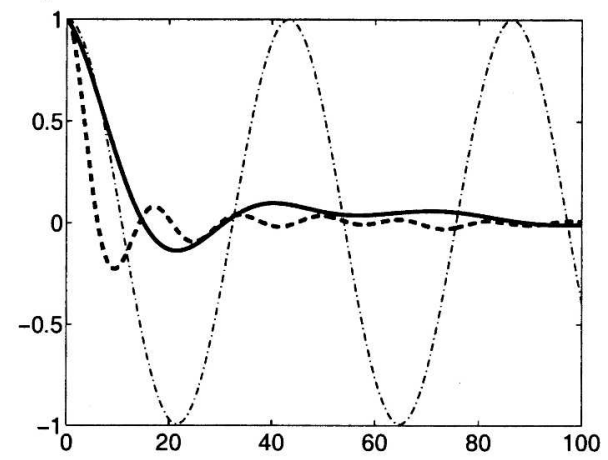

d) Mode 4

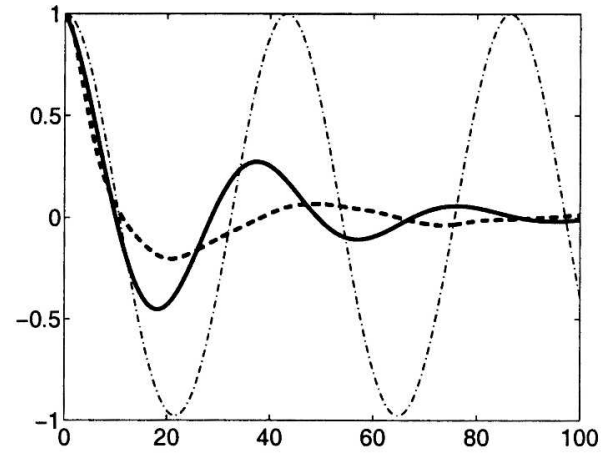

f) Mode 6

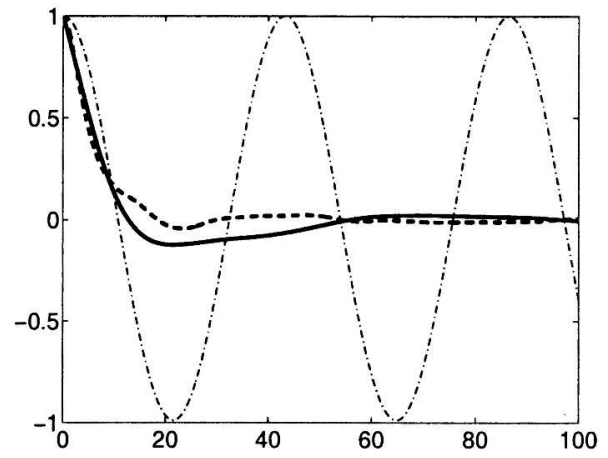

FIG. 14. Autocorrelation function for the 7-mode system. Solid line is stochastic model, dashed line is barotropic model, and dasheddotted line is bare truncation. Only diagonal correlations are used. 
a) Mode 1

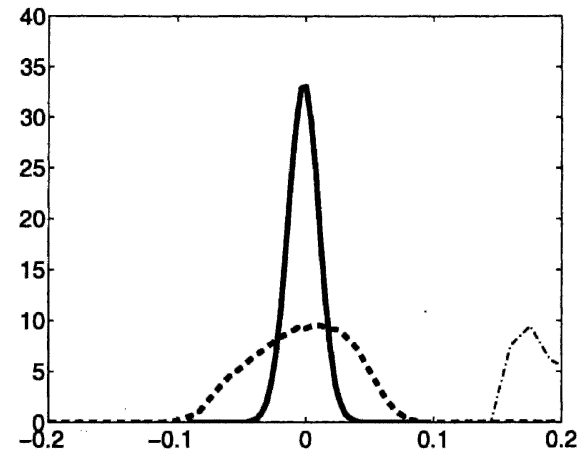

c) Mode 3

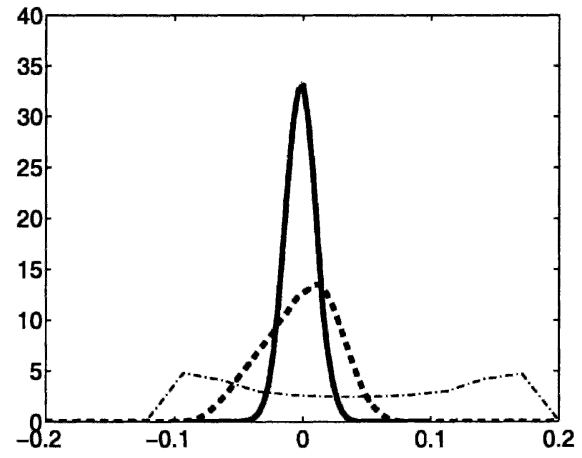

e) Mode 5

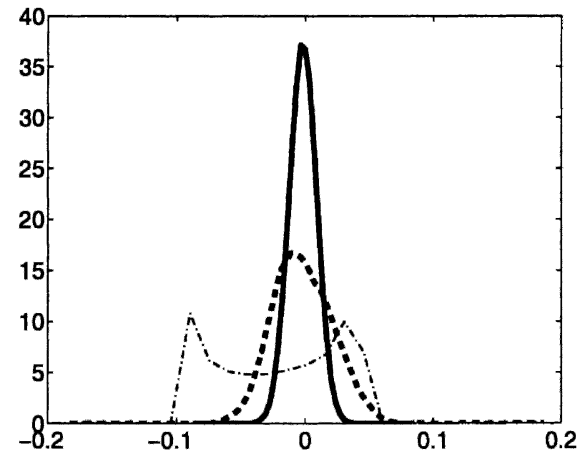

g) Mode 7

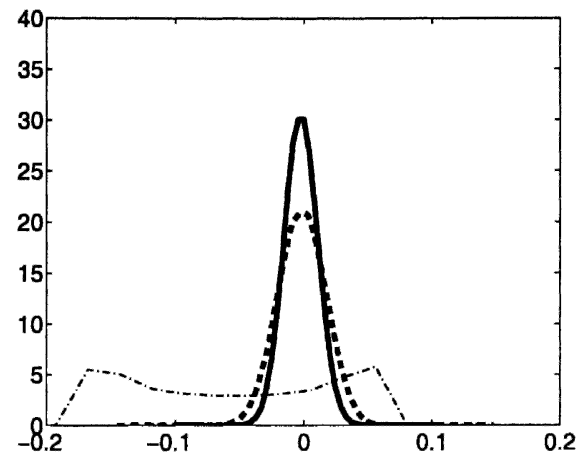

b) Mode 2

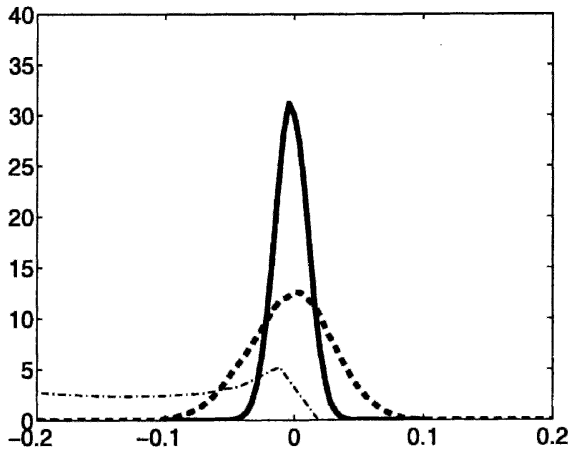

d) Mode 4

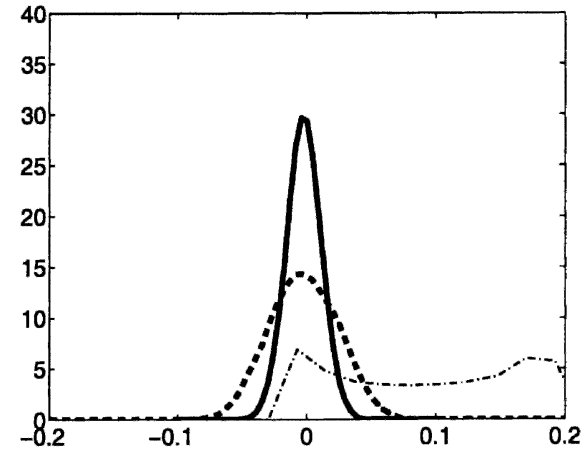

f) Mode 6

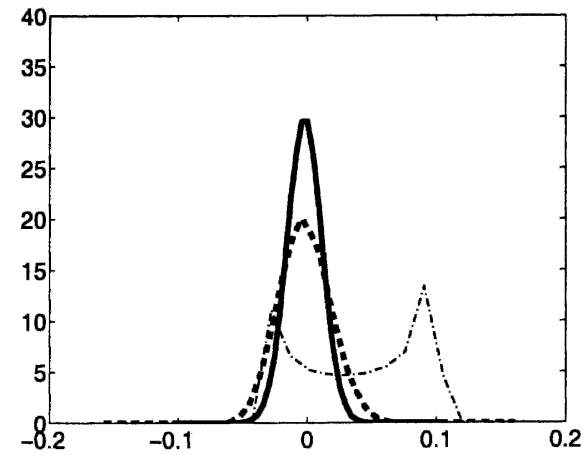

FIG. 15. PDF for the 7-mode system. Solid line is stochastic model, dashed line is barotropic model, and dashed-dotted line is bare truncation (note these figures are scaled to emphasize the stochastic and barotropic model results and not the bare truncation). The means are not subtracted. 
a) Mode 1

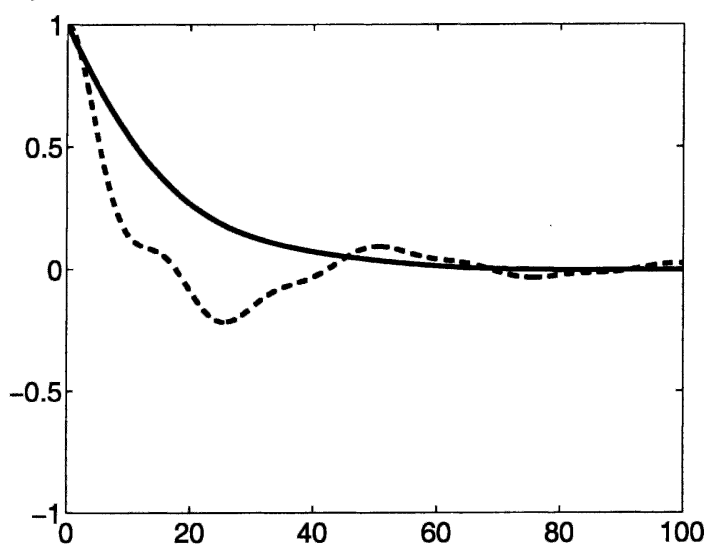

c) Mode 3

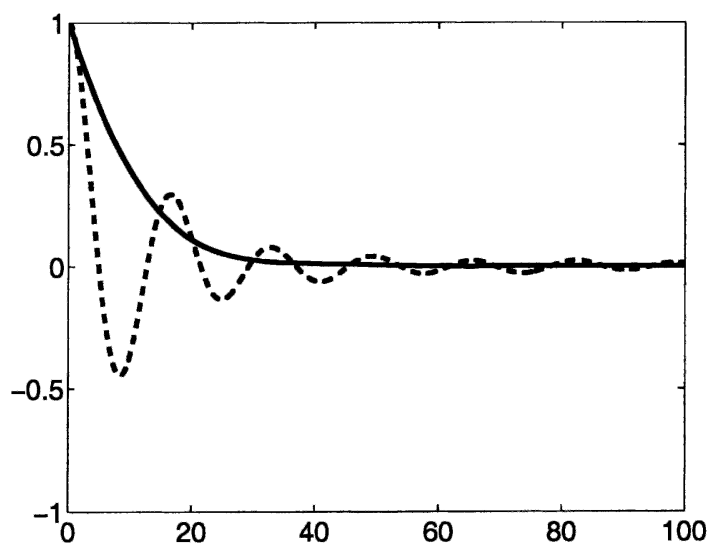

b) Mode 2

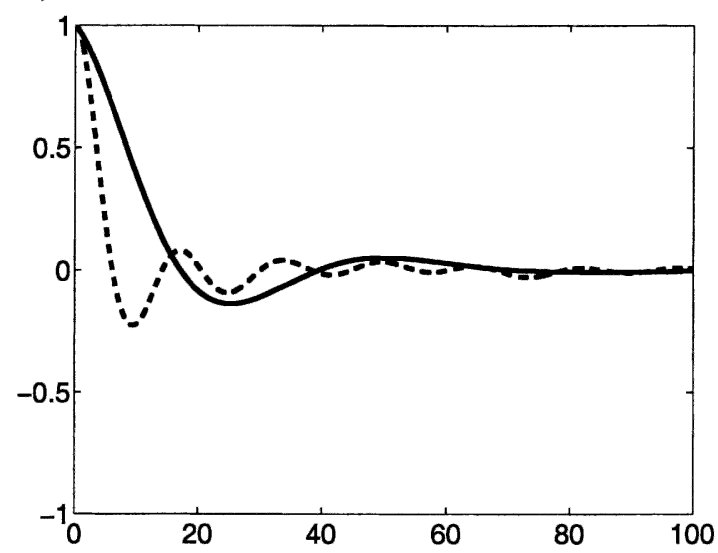

d) Mode 4

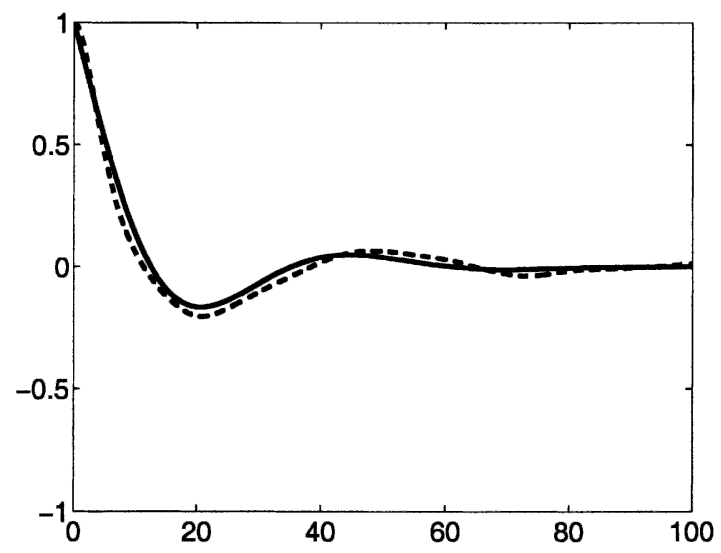

FIG. 16. Autocorrelation function for the 4-mode system with cross correlation. Solid line is stochastic model, and dashed line is barotropic model.

hand, the first 10 or 15 PIPs in the barotropic models are virtually indistinguishable from the EOFs (Kwasniok 1996). An interesting study by Farrell and Ioannou (2001) points to the potential inadequacy of EOFs as a basis for the dynamics. Their work refers to the study of the nonnormality of a stable linear operator and optimal representation of both the growing structures in the system and the structures into which these evolve. The linear operator at the climate mean state utilized here is unstable with a 14-dimensional unstable manifold unless additional ad hoc dissipation is added at the outset; thus this basis is not directly applicable here. Recently, Kwasniok (2005) has introduced a simplified PIP strategy that only utilizes tendencies in the linear dynamics but allows for instantaneous linear instabilities. Obviously, it is worthwhile to explore the basic stochastic mode reduction strategy developed here in other optimal bases besides EOFs. The authors plan to do this in a future publication.

Acknowledgments. We thank Rafail Abramov for providing us with his barotropic model code and Grant
Branstator and Daan Crommelin for their comments. The manuscript was improved by the comments of three anonymous reviewers. We acknowledge the NOAA Climate Diagnostics Center for providing us with the NCEP-NCAR reanalysis dataset. This research is funded in part through NSF-CMG Grant DMS-0222133 and NSF Grant DMS-0209959.

\section{APPENDIX A}

\section{Determination of Forcing}

The (time independent) forcing $F$ is based on observations. We are using the observed $300-\mathrm{hPa}$ vorticity and wind fields, obtained from the National Centers for Environmental Prediction (NCEP)-NCAR reanalysis data as our target climate. These data cover the years 1958-97 for the months of December-January. The initial forcing (first guess) is calculated from the NCEPNCAR dataset according to 
a) Mode 1

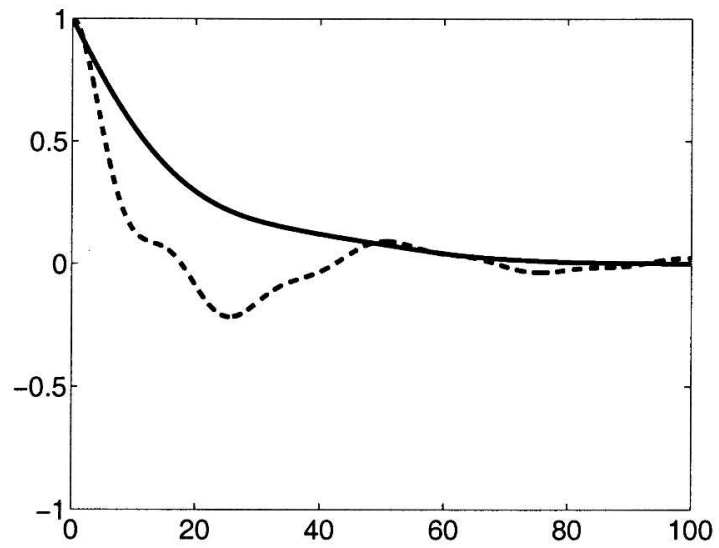

c) Mode 3

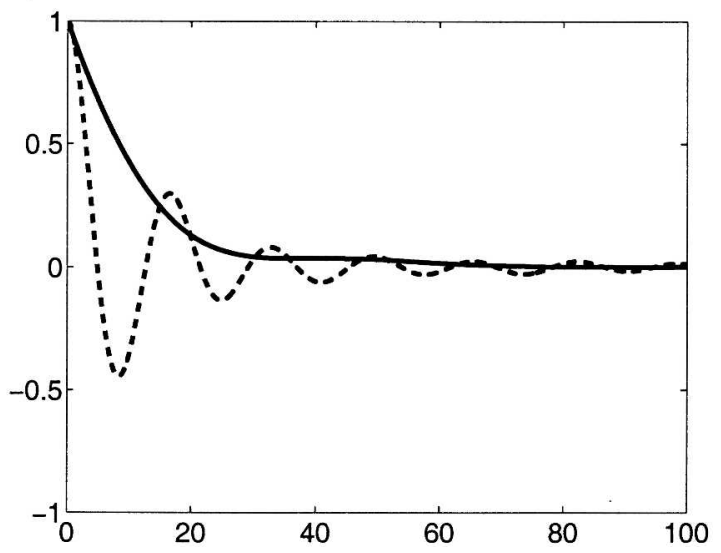

b) Mode 2

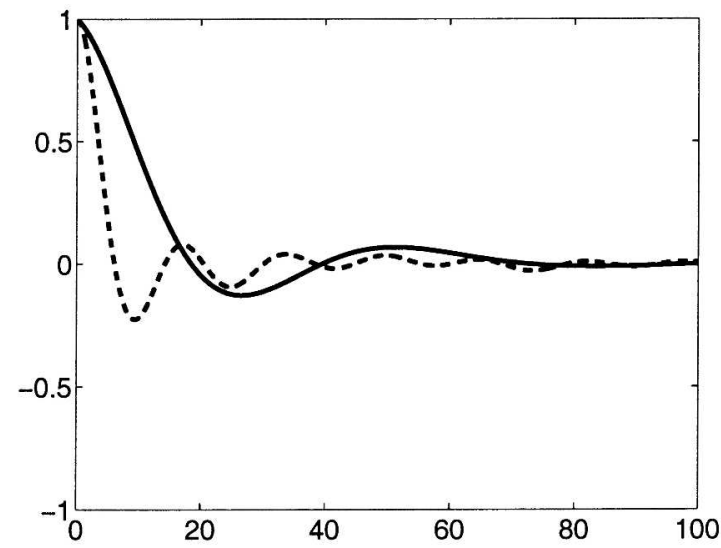

d) Mode 4

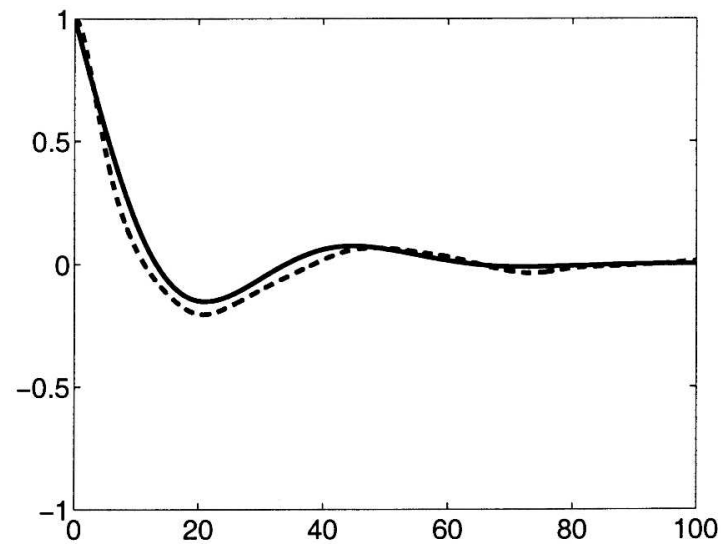

FIG. 17. Autocorrelation function for the 4-mode system consisting of only linear bare truncation and augmented linearity. Solid line is stochastic model, and dashed line is barotropic model. Only diagonal correlations are used.

$$
\begin{aligned}
\zeta_{F}(1)= & \boldsymbol{\nabla} \cdot\left[\overline{\mathbf{v}}_{\mathrm{CL}}\left(\bar{\zeta}_{\mathrm{CL}}+f+h\right)\right]+\frac{\bar{\zeta}_{\mathrm{CL}}}{\tau_{F}}+K \nabla^{6} \bar{\zeta}_{\mathrm{CL}} \\
& +\boldsymbol{\nabla} \cdot{\overline{\left(\mathbf{v}^{\prime} \zeta^{\prime}\right)}}_{\mathrm{CL}},
\end{aligned}
$$

where the prime denotes deviations from a temporal mean $\bar{X}$, and the subscript CL indicates observed climatological variables. With this forcing the model was integrated for 3600 days, and its variability has been analyzed. The standard deviation of low-pass filtered (periods $>10$ days) streamfunction is weaker and has its maximum in a different location when compared with the observations. Therefore, an iterative procedure is used to determine a forcing field $\zeta_{F}$ from $\zeta_{C L}$ and $\boldsymbol{\nabla} \cdot{\overline{\left(\mathbf{v}^{\prime} \zeta^{\prime}\right)}}_{\mathrm{CL}}$ that better matches the observations

$$
\begin{aligned}
\zeta_{F}(n+1)= & \zeta_{F}(n)+\left[\bar{\zeta}_{\mathrm{CL}}-\bar{\zeta}(n)\right]+\left[\nabla \cdot{\overline{\left(\mathbf{v}^{\prime} \zeta^{\prime}\right)}}_{\mathrm{CL}}\right. \\
& \left.-\nabla \cdot \overline{\left(\mathbf{v}^{\prime} \zeta^{\prime}\right)}(n)\right],
\end{aligned}
$$

where $n$ indicates the iteration step. For this purpose runs over 3600 days have been carried out. From these runs the actual forcing fields are calculated, and then the forcing field is updated for the next run. The reduction of the root-mean-square error equilibrates for the mean after about 30 iterations.

\section{APPENDIX B}

\section{Calculation of EOFs}

EOFs are widely used in atmospheric science. The leading EOFs extract the preferred circulation patterns of the atmosphere from a dataset. Since EOFs constitute a complete basis, the streamfunction $\psi(t)$ can be written as

$$
\psi(t)=\sum_{i} a_{i}(t) \mathbf{e}_{i},
$$

where the principal components $a_{i}(t)$ are given by the projection of $\psi(t)$ onto the EOFs $\mathbf{e}_{i}$ :

$$
a_{i}(t)=\left(\psi(t), \mathbf{e}_{i}\right),
$$

where $(\cdot, \cdot)$ denotes an inner product. To calculate EOFs that are orthogonal with respect to an inner 
product, the following eigenproblem needs to be solved:

$$
\mathrm{CMe}_{i}=\lambda_{i} \mathbf{e}_{i}
$$

where $\mathbf{M}$ is a diagonal matrix that depends on the inner product and $\mathbf{C}$ is the covariance matrix. The kinetic energy norm has the following inner product:

$$
\begin{aligned}
(\psi, \phi) & =-\frac{1}{2} \int \Delta \psi \phi d S=\frac{1}{2} \int \nabla \psi \nabla \phi d S \\
& =-\frac{1}{2} \int \psi \Delta \phi d S .
\end{aligned}
$$

Due to the representation of the spectral coefficients and by using the kinetic energy norm, the resulting covariance matrix for the eigenproblem is not necessarily symmetric. By solving a slightly different eigenproblem the covariance matrix can be made symmetric:

$$
\sqrt{\mathbf{M}} \mathbf{C} \sqrt{\mathbf{M}} \mathbf{g}_{i}=\lambda_{i} \mathbf{g}_{i} \text {. }
$$

By solving this eigenproblem the eigenvalues $\lambda_{i}$ are the same as for the original eigenproblem, only the eigenvectors $\mathbf{g}_{i}$ have to be transformed to give the eigenvectors $\mathbf{e}_{i}$ of the original problem by

$$
\mathbf{e}_{i}=(\sqrt{\mathbf{M}})^{-1} \mathbf{g}_{i} .
$$

\section{APPENDIX C}

\section{Interaction Coefficients}

Here we derive the interaction coefficients. To simplify the derivation we transform the barotropic vorticity Eq. (1) into the streamfunction equation:

$$
\frac{\partial \psi}{\partial t}=-\Delta^{-1}\left(J(\psi, \Delta \psi+f+h)-\frac{\Delta \psi}{\tau}+D \Delta^{4} \psi+F\right) .
$$

By multiplying Eq. (C1) with the EOFs and using the kinetic energy norm (appendix B) we derive the following interaction coefficients:

$$
\begin{aligned}
H_{i}= & \int \mathbf{e}_{i}\left[F-J(\bar{\psi}, f+h)-J(\bar{\psi}, \Delta \bar{\psi})-\frac{\Delta \bar{\psi}}{\tau}\right. \\
& \left.+D \Delta^{4} \bar{\psi}\right] d S, \\
L_{i j}= & -\int \mathbf{e}_{i}\left[J\left(\mathbf{e}_{j}, f+h\right)+J\left(\mathbf{e}_{j}, \Delta \bar{\psi}\right)+J\left(\bar{\psi}, \Delta \mathbf{e}_{j}\right)+\frac{\Delta \mathbf{e}_{j}}{\tau}\right. \\
& \left.-D \Delta^{4} \mathbf{e}_{j}\right] d S,
\end{aligned}
$$

and

$$
B_{i j k}=-\int \mathbf{e}_{i} J\left(\mathbf{e}_{j}, \Delta \mathbf{e}_{k}\right) d S
$$

\section{APPENDIX D}

\section{Effective Equations}

We now derive effective equations for systems of the type as given in (8) and (9). The mode elimination procedure is general but uses the following properties of the coefficients $B_{i j k}^{x y z}$ : it assumes that these coefficients are symmetric in the last two indices, that is, $B_{i j k}^{x y z}=$ $B_{i k j}^{x z y}$, and satisfy

$B_{i j k}^{x y z}+B_{j k i}^{x y z}+B_{k i j}^{x y z}=0$ (energy conservation),

$$
\sum_{i j k} B_{i j k}^{x y z} \frac{\partial}{\partial x_{i}} y_{j} z_{k}=0 \text { (Liouville property), }
$$

where $x, y$, and $z$ each stand for $\alpha$ or $\beta$.

To derive effective equations for the slow modes $\alpha$, we closely follow the mode elimination procedure used and developed in MTV99, MTV01, MTV02, MTV03, but we give a more seamless version of the results in these papers, which is more convenient for the computation of the coefficients in the effective equations. The mode elimination procedure is based on the assumption that the fastest component of the dynamics of the fast modes in Eq. (9); that is, the dynamical system

$$
\dot{c}_{i}=\sum_{j k} B_{i j k}^{\beta \beta \beta} c_{j} c_{k}
$$

is ergodic and mixing with integrable decay of correlation. In other words, we assume that for almost all initial conditions, and suitable functions $f$ and $g$, we have

$$
\lim _{T \rightarrow \infty} \frac{1}{T} \int_{0}^{T} f(c(t)) d t=\langle f\rangle
$$

where $\langle\cdot\rangle$ denotes expectation with respect to some appropriate invariant distribution, and

$$
\begin{aligned}
G(s)= & \lim _{T \rightarrow \infty} \frac{1}{T} \int_{0}^{T} g(c(t+s), c(t)) d t \\
& -\lim _{T \rightarrow \infty} \frac{1}{T^{2}} \int_{0}^{T} \int_{0}^{T} g\left(c(t), c\left(t^{\prime}\right)\right) d t d t^{\prime}
\end{aligned}
$$

is an integrable function of $s$; that is, $\left|\int_{0}^{\infty} G(s) d s\right|<\infty$. Under the above assumptions, it can be shown (Kurtz 1973; Papanicolaou 1976) that in the limit as $\varepsilon \rightarrow 0$ the dynamics of the slow modes $\alpha_{i}$ in Eq. (9) can be represented by the following effective Itô stochastic differential equation (SDE):

$$
\begin{aligned}
d \alpha_{i}(t)= & \sum_{j} L_{i j}^{\alpha \alpha} \alpha_{j}(t) d t+\sum_{j k} B_{i j k}^{\alpha \alpha \alpha} \alpha_{j}(t) \alpha_{k}(t) d t \\
& +G_{i} d t+\sqrt{2} \sum_{j} \sigma_{i j}(\alpha(t)) d W_{i} .
\end{aligned}
$$


The second line in this equation consists of the terms that emerge to represent the effect of the fast modes on the slow ones as $\varepsilon \rightarrow 0$. Here $W=\left(W_{1}, \cdots, W_{m}\right)$ is an $m$-dimensional Wiener process, and $G_{i}$ is given by

$$
G_{i}=\lim _{T \rightarrow \infty} \frac{1}{T} \int_{0}^{T} d t \int_{0}^{\infty} d s K_{j}^{\beta}[c(t)] \sum_{j}\left\{\frac{\partial}{\partial c_{j}(t)} K_{i}^{\alpha}[c(t+s)]\right\}+\lim _{T \rightarrow \infty} \frac{1}{T} \int_{0}^{T} d t \int_{0}^{\infty} d s \sum_{j} K_{j}^{\alpha}[c(t)]\left\{\frac{\partial}{\partial \alpha_{j}} K_{i}^{\alpha}[c(t+s)]\right\},
$$

$\sigma_{i j}$ is the Cholesky decomposition (i.e., $P_{i j}=\Sigma_{k} \sigma_{i k} \sigma_{j k}$ ) of

$$
P_{i j}=\lim _{T \rightarrow \infty} \frac{1}{T} \int_{0}^{T} d t \int_{0}^{\infty} d s K_{i}^{\alpha}(c(t)) K_{j}^{\alpha}(c(t+s)),
$$

and

$$
\begin{aligned}
K_{i}^{\alpha}(c)= & \sum_{j} L_{i j}^{\alpha \beta} c_{j}+2 \sum_{j k} B_{i j k}^{\alpha \alpha \beta} \alpha_{j} c_{k}+\sum_{j k} B_{i j k}^{\alpha \beta \beta} c_{j} c_{k}, \\
K_{i}^{\beta}(c)= & \sum_{j} L_{i j}^{\beta \alpha} \alpha_{j}+\sum_{j} L_{i j}^{\beta \beta} c_{j}+2 \sum_{j k} B_{i j k}^{\beta \alpha \beta} \alpha_{j} c_{k} \\
& +\sum_{j k} B_{i j k}^{\beta \alpha \alpha} \alpha_{j} \alpha_{k} .
\end{aligned}
$$

The sequel of this appendix is to put the SDE (D6) in a form more suitable for computations. To do so, we shall make the following assumptions:

1) The statistics of $c(t)$ solution of (D3) can be approximated by the statistics of $\beta(t)$ solution of original system in (9). This means all the $c$ can be replaced by $\beta$ in (D7), (D8), and (D9).

2) The moments of $\beta(t)$ can be approximated as if $\beta(t)$ were a Gaussian process. This means in particular that moments of order 3 vanish, whereas moments of order 4 can be related to moment of order 2 as

$$
\begin{aligned}
\lim _{T \rightarrow \infty} & \frac{1}{T} \int_{0}^{T} d t \beta_{i}(t) \beta_{j}(t) \beta_{k}(t+s) \beta_{l}(t+s) \\
& =\mathcal{B}_{i} \mathcal{B}_{k} \delta_{i j} \delta_{k l}+\mathcal{B}_{i k}(s) \mathcal{B}_{j l}(s)+\mathcal{B}_{i l}(s) \mathcal{B}_{j k}(s),
\end{aligned}
$$

where

$$
\mathcal{B}_{i j}(s)=\mathcal{B}_{j i}(-s)=\lim _{T \rightarrow \infty} \frac{1}{T} \int_{0}^{T} d t \beta_{i}(t) \beta_{j}(t+s)
$$

and

$$
\mathcal{B}_{i j}(0)=\mathcal{B}_{i} \delta_{i j} \text {. }
$$

Under these assumptions, straightforward manipulations show that the drift $G_{i}$ and diffusion tensor $H_{i j}$ reduce to

$$
\begin{aligned}
& G_{i}=\tilde{H}_{i}+\sum_{j} \tilde{L}_{i j} \alpha_{j}+\sum_{j k} \tilde{B}_{i j k} \alpha_{j} \alpha_{k}+\sum_{j k l} \tilde{M}_{i j k l} \alpha_{j} \alpha_{k} \alpha_{l}, \\
& P_{i j}=Q_{i j}+\sum_{k} U_{i j k} \alpha_{k}+\sum_{k l} V_{i j k l} \alpha_{k} \alpha_{l} .
\end{aligned}
$$

The new forcing coefficients are given by

$$
\tilde{H}_{i}=\tilde{H}_{i}^{(1)}+\tilde{H}_{i}^{(2)}
$$

with

$$
\begin{aligned}
& \tilde{H}_{i}^{(1)}=\sum_{j k l} \int_{-\infty}^{\infty} d t L_{j l}^{\alpha \beta} B_{i j k}^{\alpha \beta \beta} \mathcal{B}_{l k}(t), \\
& \tilde{H}_{i}^{(2)}=\sum_{k l m n} \int_{-\infty}^{\infty} d t L_{k l}^{\beta \beta} B_{i m n}^{\alpha \beta \beta} \frac{\mathcal{B}_{k m}(t) \mathcal{B}_{l n}(t)+\mathcal{B}_{k n}(t) \mathcal{B}_{l m}(t)}{\mathcal{B}_{k}} .
\end{aligned}
$$

The new linear interaction coefficients are given by

$$
\tilde{L}_{j i}=\tilde{L}_{j i}^{(1)}+\tilde{L}_{j i}^{(2)}+\tilde{L}_{j i}^{(3)}
$$

with

$$
\begin{aligned}
\tilde{L}_{i j}^{(1)}= & \frac{1}{2} \sum_{k l} \int_{-\infty}^{\infty} d t L_{k j}^{\beta \alpha} L_{i l}^{\alpha \beta} \frac{\mathcal{B}_{k l}(t)}{\mathcal{B}_{k}}, \\
\tilde{L}_{i j}^{(2)}= & \frac{1}{2} \sum_{k l m n} \int_{-\infty}^{\infty} d t B_{i m n}^{\alpha \beta \beta}\left(\frac{B_{l j k}^{\beta \alpha \beta}}{\mathcal{B}_{l}}+\frac{B_{k j l}^{\beta \alpha \beta}}{\mathcal{B}_{k}}\right) \\
& {\left[\mathcal{B}_{k m}(t) \mathcal{B}_{l n}(t)+\mathcal{B}_{k n}(t) \mathcal{B}_{l m}(t)\right], } \\
\tilde{L}_{i j}^{(3)}= & 2 \sum_{k l n} \int_{-\infty}^{\infty} d t B_{k j l}^{\alpha \alpha \beta} B_{i k n}^{\alpha \alpha \beta} \mathcal{B}_{l n}(t) .
\end{aligned}
$$

The new quadratic nonlinear interaction coefficients are given by

$$
\tilde{B}_{j i k}=\tilde{B}_{j i k}^{(1)}+\tilde{B}_{j i k}^{(2)}
$$

with

$$
\begin{aligned}
\tilde{B}_{i j k}^{(1)} & =\frac{1}{2} \sum_{m n} \int_{-\infty}^{\infty} d t B_{m j k}^{\beta \alpha \alpha} L_{i n}^{\alpha \beta} \frac{\mathcal{B}_{m n}(t)}{\mathcal{B}_{m}}, \\
\tilde{B}_{i j k}^{(2)} & =\frac{1}{2} \sum_{m n} \int_{-\infty}^{\infty} d t\left(L_{m j}^{\beta \alpha} B_{i k n}^{\alpha \alpha \beta}+L_{m k}^{\beta \alpha} B_{i j n}^{\alpha \alpha \beta}\right) \frac{\mathcal{B}_{m n}(t)}{\mathcal{B}_{m}} .
\end{aligned}
$$

The new cubic nonlinear interaction coefficients are given by 


$$
\begin{aligned}
\tilde{M}_{i j k l}= & \frac{1}{3} \sum_{m n} \int_{-\infty}^{\infty} d t\left(B_{n k l}^{\beta \alpha \alpha} B_{i j m}^{\alpha \alpha \beta}+B_{n l j}^{\beta \alpha \alpha} B_{i k m}^{\alpha \alpha \beta}\right. \\
& \left.+B_{n j k}^{\beta \alpha \alpha} B_{i l m}^{\alpha \alpha \beta}\right) \frac{\mathcal{B}_{n m}(t)}{\mathcal{B}_{n}} .
\end{aligned}
$$

The diffusion coefficients are given by

$$
Q_{i j}=Q_{i j}^{(1)}+Q_{i j}^{(2)}
$$

with

$$
\begin{aligned}
Q_{i j}^{(1)}= & \frac{1}{2} \sum_{l k} \int_{-\infty}^{\infty} d t L_{i l}^{\alpha \beta} L_{j k}^{\alpha \beta} \mathcal{B}_{l k}(t) \\
Q_{i j}^{(2)}= & \frac{1}{2} \sum_{k l m n} \int_{-\infty}^{\infty} d t B_{i k l}^{\alpha \beta \beta} B_{j m n}^{\alpha \beta \beta} \\
& {\left[\mathcal{B}_{k m}(t) \mathcal{B}_{l n}(t)+\mathcal{B}_{k n}(t) \mathcal{B}_{l m}(t)\right] }
\end{aligned}
$$

and

$$
\begin{aligned}
U_{i j k} & =\sum_{l n} \int_{-\infty}^{\infty} d t\left(L_{i l}^{\alpha \beta} B_{j k n}^{\alpha \alpha \beta}+L_{j l}^{\alpha \beta} B_{i k n}^{\alpha \alpha \beta}\right) \mathcal{B}_{l n}(t) \\
V_{j i k l} & =2 \sum_{m n} \int_{-\infty}^{\infty} d t B_{i k m}^{\alpha \alpha \beta} B_{j l n}^{\alpha \alpha \beta} \mathcal{B}_{m n}(t) .
\end{aligned}
$$

The terms involving $H_{i}^{(1)}$ and $L_{i j}^{(3)}$ are Itô drifts.

Note that the effective equations in (D6) can be put in the form

$$
\begin{aligned}
d \alpha_{i}(t)= & \sum_{j} L_{i j}^{\alpha \alpha} \alpha_{j}(t) d t+\sum_{j k} B_{i j k}^{\alpha \alpha \alpha} \alpha_{j}(t) \alpha_{k}(t) d t+G_{i} d t \\
& +\sqrt{2} \sum_{j} \sigma_{i j}^{(1)}[\alpha(t)] d W_{i}^{(1)}+\sqrt{2} \sum_{j} \sigma_{i j}^{(2)} d W_{i}^{(2)},
\end{aligned}
$$

where $W^{(1)}=\left(W_{1}^{(1)}, \cdots, W_{m}^{(1)}\right)$ and $W^{(2)}=\left(W_{1}^{(2)}, \cdots\right.$, $\left.W_{m}^{(2)}\right)$ are two independent $m$-dimensional Wiener processes and $\sigma_{j i}^{(1)}, \sigma_{j i}^{(2)}$ are defined so that

$$
\begin{aligned}
Q_{i j}^{(1)}+\sum_{k} U_{i j k} \alpha_{k}(t) & \\
+\sum_{k} \sum_{l} V_{i j k l} \alpha_{k}(t) \alpha_{l}(t) & =\sum_{k} \sigma_{i k}^{(1)}[\alpha(t)] \sigma_{j k}^{(1)}[\alpha(t)], \\
Q_{i j}^{(2)} & =\sum_{k} \sigma_{i k}^{(2)} \sigma_{j k}^{(2)} .
\end{aligned}
$$

It is straightforward to verify that the left-hand sides in Eq. (D34) are positive definite $m \times m$ tensors, so the tensors $\sigma_{i j}^{(1)}[\alpha(t)]$ and $\sigma_{i j}^{(2)}$ exist and can be calculated by Cholesky decomposition.

\section{REFERENCES}

Achatz, U., and G. W. Branstator, 1999: A two-layer model with empirical linear corrections and reduced order for studies of internal climate variability. J. Atmos. Sci., 56, 3140-3160.

Barnston, A. G., and R. E. Livezey, 1987: Classification, season- ality, and persistence of low-frequency atmospheric circulation patterns. Mon. Wea. Rev., 115, 1083-1126.

Berner, J., 2003: Detection and stochastic modeling of nonlinear signatures in the geopotential height field of an atmospheric general circulation model. Ph.D. thesis, Universität Bonn, $157 \mathrm{pp}$.

Branstator, G. W., and S. E. Haupt, 1998: An empirical model of barotropic atmospheric dynamics and its response to tropical forcing. J. Climate, 11, 2645-2667.

Charney, J. G., and J. G. DeVore, 1979: Multiple flow equilibria in the atmosphere and blocking. J. Atmos. Sci., 36, 1205-1216.

Crommelin, D. T., and A. J. Majda, 2004: Strategies for model reduction: Comparing different optimal bases. J. Atmos. Sci., 61, 2206-2217.

Delsole, T., 2001: Optimally persistent patterns in time-varying fields. J. Atmos. Sci., 58, 1341-1356.

_ 2004: Stochastic models of quasigeostrophic turbulence. Surv. Geophys., 25, 107-149.

Farrell, B. F., and P. J. Ioannou, 2001: Accurate low-dimensional approximation of the linear dynamics of fluid flow. J. Atmos. Sci., 58, 2771-2789.

Feldstein, S. B., 2000: The timescale, power spectra, and climate noise properties of teleconnection patterns. J. Climate, 13, 4430-4440.

Gardiner, C. W., 1985: Handbook of Stochastic Methods. Springer-Verlag, $442 \mathrm{pp}$.

Hasselmann, K., 1988: PIPs and POPs: The reduction of complex dynamical systems using principal interaction and oscillation patterns. J. Geophys. Res., 93, 11 015-11 021.

Kurtz, T. G., 1973: A limit theorem for perturbed operator semigroups with applications to random evolutions. J. Funct. Anal., 12, 55-67.

Kwasniok, F., 1996: The reduction of complex dynamical systems using principal interaction patterns. Physica D, 92, 28-60.

—, 2004: Empirical low-order models of barotropic flow. J. Atmos. Sci., 61, 235-245.

Legras, B., and M. Ghil, 1985: Persistent anomalies, blocking and variations in atmospheric predictability. J. Atmos. Sci., 42, 433-471.

Majda, A. J., and I. Timofeyev, 2000: Remarkable statistical behavior for truncated Burgers-Hopf dynamics. Proc. Natl. Acad. Sci. U.S.A., 97, 12 413-12 417.

, and _ 2004: Low dimensional chaotic dynamics versus intrinsic stochastic chaos: A paradigm model. Physica D, 199, 339-368.

—,- , and E. Vanden-Eijnden, 1999: Models for stochastic climate prediction. Proc. Natl. Acad. Sci. U.S.A., 96, 14687 14691.

- _ - and 2001: A mathematical framework for stochastic climate models. Commun. Pure Appl. Math., 54, 891974.

— - , and — 2002: A priori tests of a stochastic mode reduction strategy. Physica $D, \mathbf{1 7 0}, 206-252$.

$\longrightarrow,-$, and $\_$2003: Systematic strategies for stochastic mode reduction in climate. J. Atmos. Sci., 60, 1705-1722.

Marshall, J., and Coauthors, 2001: North Atlantic climate variability: Phenomena, impacts and mechanisms. Int. J. Climatol., 21, 1863-1898.

Newman, M., P. D. Sardeshmukh, and C. Penland, 1997: Stochastic forcing of the wintertime extratropical flow. J. Atmos. Sci., $\mathbf{5 4}, 435-455$.

Papanicolaou, G. C., 1976: Some probalistic problems and methods in singular perturbations. Rocky Mountain J. Math., 6, 653-674.

Percival, D. B., J. E. Overland, and H. O. Mofjeld, 2001: Interpretation of North Pacific variability as a short- and longmemory process. J. Climate, 14, 4545-4559.

Reinhold, B. B., and R. T. Pierrehumbert, 1982: Dynamics of 
weather regimes: Quasi-stationary waves and blocking. Mon. Wea. Rev., 110, 1105-1145.

Schubert, S. D., 1985: A statistical-dynamical study of empirically determined modes of atmospheric variability. J. Atmos. Sci., 42, 3-17.

Selten, F. M., 1995: An efficient description of the dynamics of barotropic flow. J. Atmos. Sci., 52, 915-936.

Stephenson, D. B., V. Pavan, and R. Bojariu, 2000: Is the North Atlantic Oscillation a random walk? Int. J. Climatol., 20, $1-18$.

Thompson, D. W. J., and J. M. Wallace, 1998: The Arctic Oscillation signature in the wintertime geopotential height and temperature fields. Geophys. Res. Lett., 25, 1297-1300.

—, and —, 2001: Regional climate impacts of the Northern Hemisphere annular mode and associated climate trends. Science, 293, 85-89.

Wallace, J. M., and D. S. Gutzler, 1981: Teleconnections in the geopotential height field during the Northern Hemisphere winter. Mon. Wea. Rev., 109, 784-804.

- and D. W. J. Thompson, 2002: The Pacific center of action of the Northern Hemisphere annular mode: Real or artifact? J. Climate, 15, 1987-1991.

Whitaker, J. S., and P. D. Sardeshmukh, 1998: A linear theory of extratropical synoptic eddy statistics. J. Atmos. Sci., 55, 237 258.

Winkler, C. R., M. Newman, and P. D. Sardeshmukh, 2001: A linear model of wintertime low-frequency variability. Part I: Formulation and forecast skill. J. Climate, 14, 4474-4494.

Wunsch, C., 1999: The interpretation of short climate records, with comments on the North Atlantic and Southern Oscillations. Bull. Amer. Meteor. Soc., 80, 245-255.

Zhang, Y., and I. M. Held, 1999: A linear stochastic model of a GCM's midlatitude storm tracks. J. Atmos. Sci., 56, 34163435 . 